\title{
Front Matter: Volume 9145
}

, "Front Matter: Volume 9145," Proc. SPIE 9145, Ground-based and Airborne Telescopes V, 914501 (5 September 2014); doi: 10.1117/12.2075023

SPIE Event: SPIE Astronomical Telescopes + Instrumentation, 2014, Montréal, Quebec, Canada 


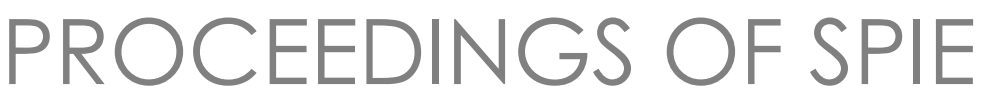

\title{
Ground-based and Airborne Telescopes V
}

\author{
Larry M. Stepp \\ Roberto Gilmozzi \\ Helen J. Hall \\ Editors
}

\section{2-27 June 2014}

Montréal, Canada

Sponsored by

SPIE

Cooperating Organizations

American Astronomical Society (United States) • Australian Astronomical Observatory (Australia) • Association of Universities for Research in Astronomy (AURA) • Canadian Astronomical Society (CASCA) (Canada) • Canadian Space Agency (Canada) • European Astronomical Society (Switzerland) • European Southern Observatory (Germany) • National Radio Astronomy Observatory • Royal Astronomical Society (United Kingdom) • Science \& Technology Facilities Council (United Kingdom)

Published by

SPIE 
The papers included in this volume were part of the technical conference cited on the cover and title page. Papers were selected and subject to review by the editors and conference program committee. Some conference presentations may not be available for publication. The papers published in these proceedings reflect the work and thoughts of the authors and are published herein as submitted. The publisher is not responsible for the validity of the information or for any outcomes resulting from reliance thereon.

Please use the following format to cite material from this book:

Author(s), "Title of Paper," in Ground-based and Airborne Telescopes V, edited by Larry M. Stepp, Roberto Gilmozzi, Helen J. Hall, Proceedings of SPIE Vol. 9145 (SPIE, Bellingham, WA, 2014) Article CID Number.

ISSN: 0277-786X

ISBN: 9780819496133

Published by

SPIE

P.O. Box 10, Bellingham, Washington 98227-0010 USA

Telephone +1 3606763290 (Pacific Time) · Fax +1 3606471445

SPIE.org

Copyright @ 2014, Society of Photo-Optical Instrumentation Engineers.

Copying of material in this book for internal or personal use, or for the internal or personal use of specific clients, beyond the fair use provisions granted by the U.S. Copyright Law is authorized by SPIE subject to payment of copying fees. The Transactional Reporting Service base fee for this volume is $\$ 18.00$ per article (or portion thereof), which should be paid directly to the Copyright Clearance Center (CCC), 222 Rosewood Drive, Danvers, MA 01923. Payment may also be made electronically through CCC Online at copyright.com. Other copying for republication, resale, advertising or promotion, or any form of systematic or multiple reproduction of any material in this book is prohibited except with permission in writing from the publisher. The CCC fee code is 0277-786X/14/\$18.00.

Printed in the United States of America.

Publication of record for individual papers is online in the SPIE Digital Library.

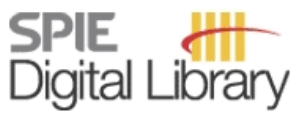

SPIEDigitalLibrary.org

Paper Numbering: Proceedings of SPIE follow an e-First publication model, with papers published first online and then in print and on CD-ROM. Papers are published as they are submitted and meet publication criteria. A unique, consistent, permanent citation identifier (CID) number is assigned to each article at the time of the first publication. Utilization of CIDs allows articles to be fully citable as soon as they are published online, and connects the same identifier to all online, print, and electronic versions of the publication. SPIE uses a six-digit CID article numbering system in which:

- The first four digits correspond to the SPIE volume number.

- The last two digits indicate publication order within the volume using a Base 36 numbering

system employing both numerals and letters. These two-number sets start with 00, 01, 02, 03, 04, 05, 06, 07, 08, 09, 0A, 0B ... 0Z, followed by 10-1Z, 20-2Z, etc.

The CID Number appears on each page of the manuscript. The complete citation is used on the first page, and an abbreviated version on subsequent pages. Numbers in the index correspond to the last two digits of the six-digit CID Number. 


\title{
Contents
}

\section{Part One}

\author{
xxxv Conference Committee \\ xxxix Introduction
}

\section{PROJECT REVIEWS I}

914502 The Large Binocular Telescope: binocular all the time [9145-1]

J. M. Hill, D. S. Ashby, J. G. Brynnel, J. C. Christou, J. K. Little, D. M. Summers, C. Veillet, R. M. Wagner, Large Binocular Telescope Observatory, The Univ. of Arizona (United States)

914503 Ideas for future large single dish radio telescopes [9145-177]

H. J. Kärcher, MT Mechatronics GmbH (Germany); J. W. M. Baars, Max-Planck-Institut für Radioastronomie (Germany)

914504 Solaris: a global network of autonomous observatories in the southern hemisphere [9145-3] S. K. Kozłowski, P. Sybilski, Nicolaus Copernicus Astronomical Ctr. (Poland); M. Konacki, Nicolaus Copernicus Astronomical Ctr. (Poland) and Astronimcal Observatory, Adam Mickewicz Univ. (Poland); R. K. Pawłaszek, M. Ratajczak, Nicolaus Copernicus Astronomical Ctr. (Poland); K. G. Helminiak, Subaru Telescope, National Astronomical Observatory of Japan (United States) and Nicolaus Copernicus Astronomical Ctr. (Poland)

914506 Deployment of the Hobby-Eberly Telescope wide field upgrade [9145-5]

G. J. Hill, McDonald Observatory, The Univ. of Texas at Austin (United States) and The Univ. of Texas at Austin (United States); N. Drory, J. Good, H. Lee, B. L. Vattiat, McDonald Observatory, The Univ. of Texas at Austin (United States); H. Kriel, McDonald Observatory, The Univ. of Texas at Austin (United States) and Hobby-Eberly Telescope, The Univ. of Texas at Austin (United States); R. Bryant, Hobby-Eberly Telescope, The Univ. of Texas at Austin (United States); L. Elliot, M. Landriau, R. Leck, D. Perry, J. Ramsey, R. Savage, McDonald Observatory, The Univ. of Texas at Austin (United States); R. D. Allen, Texas A\&M Univ. (United States); G. Damm, Hobby-Eberly Telescope, The Univ. of Texas at Austin (United States); D. L. DePoy, Texas A\&M Univ. (United States); J. Fowler, Hobby-Eberly Telescope, The Univ. of Texas at Austin (United States); K. Gebhardt, The Univ. of Texas at Austin (United States); M. Haeuser, Texas A\&M Univ. (United States); P. MacQueen, McDonald Observatory, The Univ. of Texas at Austin (United States); J. L. Marshall, Texas A\&M Univ. (United States); J. Martin, Hobby-Eberly Telescope, The Univ. of Texas at Austin (United States); T. Prochaska, Texas A\&M Univ. (United States); L. W. Ramsey, Univ.-Sternwarte München (Germany); J.-P. Rheault, Texas A\&M Univ. (United States); M. Shetrone, E. Schroeder Mrozinski, Hobby-Eberly Telescope, The Univ. of Texas at Austin (United States); S. E. Tuttle, McDonald Observatory, The Univ. of Texas at Austin (United States); M. E. Cornell, MIT Lincoln Lab. (United States); J. Booth, McDonald Observatory, The Univ. of Texas at Austin (United States); W. Moreira, The Univ. of Texas at Austin (United States) 
914507 Overview of University of Tokyo Atacama Observatory 6.5m telescope project (Invited Paper) [9145-6]

Y. Yoshii, M. Doi, K. Kohno, T. Miyata, K. Motohara, K. Kawara, M. Tanaka, T. Minezaki, S. Sako, T. Morokuma, Y. Tamura, T. Tanabe, H. Takahashi, M. Konishi, T. Kamizuka, Institute of Astronomy, The Univ. of Tokyo (Japan); S. Koshida, Institute of Astronomy, The Univ. of Tokyo (Japan) and Pontificia Univ. Católica de Chile (Chile); N. Kato, Institute of Astronomy, The Univ. of Tokyo (Japan); T. Aoki, T. Soyano, K. Tarusawa, Kiso Observatory, The Univ. of Tokyo (Japan); T. Handa, Kagoshima Univ. (Japan); L. Bronfman, M. T. Ruiz, M. Hamuy, R. Mendez, Univ. de Chile (Chile)

914508 The Cherenkov Telescope Array single-mirror small size telescope project: status and prospects [9145-111]

J. A. Aguilar, Univ. de Genève (Switzerland); W. Bilnik, AGH Univ. of Science and Technology (Poland); L. Bogacz, Jagiellonian Univ. (Poland); T. Bulik, Astronomical Observatory, Warsaw Univ. (Poland); A. Christov, D. della Volpe, Univ. de Genève (Switzerland); M. Dyrda, Institute of Nuclear Physics (Poland); A. Frankowski, Nicolaus Copernicus Astronomical Ctr. (Poland); M. Grudzińska, Astronomical Observatory, Warsaw Univ. (Poland); J. Grygorczuk, Space Research Ctr. (Poland); M. Heller, Univ. de Genève (Switzerland); B. Idźkowski, Jagiellonian Univ. (Poland); M. Janiak, Nicolaus Copernicus Astronomical Ctr. (Poland); M. Jamrozy, Jagiellonian Univ. (Poland); M. Karczewski, Space Research Ctr. (Poland); J. Kasperek, AGH Univ. of Science and Technology (Poland); E. Lyard, Univ. de Genève (Switzerland); A. Marszałek, Astronomical Observatory, Jagiellonian Univ. (Poland); J. Michałowski, Institute of Nuclear Physics (Poland); M. Rameez, Univ. de Genève (Switzerland); R. Moderski, Nicolaus Copernicus Astronomical Ctr. (Poland); T. Montaruli, A. Neronov, Univ. de Genève (Switzerland); J. Nicolau-Kukliński, Space Research Ctr. (Poland); J. Niemiec, Institute of Nuclear Physics (Poland); M. Ostrowski, Jagiellonian Univ. (Poland); P. Paśko, Ł. Płatos, Space Research Ctr. (Poland); E. Prandini, Univ. de Genève (Switzerland); J. Rafalski, Institute of Nuclear Physics (Poland); P. J. Rajda, AGH Univ. of Science and Technology (Poland); M. Rataj, Space Research Ctr. (Poland); M. Rupiński, AGH Univ. of Science and Technology (Poland); K. Rutkowski, K. Seweryn, M. Sidz, Space Research Ctr. (Poland); Ł. Stawarz, M. Stodulska, Jagiellonian Univ. (Poland); M. Stodulski, Institute of Nuclear Physics (Poland); M. Tokarz, Space Research Ctr. (Poland); S. Toscano, I. Troyano Pujadas, R. Walter, Univ. de Genève (Switzerland); P. Wawer, R. Wawrzaszek, L. Wiśniewski, Space Research Ctr. (Poland); K. Winiarski, AGH Univ. of Science and Technology (Poland); K. Ziętara, Astronomical Observatory, Jagiellonian Univ. (Poland); P. Ziółkowski, P. Źychowski, Institute of Nuclear Physics (Poland)

UPGRADES TO EXISTING OBSERVATORIES

914509 Development of a wide field spherical aberration corrector for the Hobby Eberly Telescope: design fabrication and alignment [9145-8]

C. J. Oh, E. Frater, A. E. Lowman, P. Su, C. Zhao, P. Zhou, J. H. Burge, College of Optical Sciences, The Univ. of Arizona (United States) 
$91450 \mathrm{~A}$ Conceptual design study to determine optimal enclosure vent configuration for the Maunakea Spectroscopic Explorer (MSE) [9145-9]

K. Szeto, NRC - Herzburg Institute of Astrophysics (Canada); K. Vogiatzis, Thirty Meter Telescope Observatory Corp. (United States); H. Hangan, C. M. Jubayer, WindEEE Research Institute (Canada); C. Breckenridge, N. Loewen, Dynamic Structures Ltd. (Canada); S. Bauman, D. Salmon, Canada-France-Hawaii Telescope (United States)

9145 OB The RadioAstron Green Bank Earth Station [9145-10]

H. A. Ford, R. Anderson, National Radio Astronomy Observatory (United States);

K. Belousov, Astro Space Ctr. of Lebedev Physical Institute (Russian Federation);

J. J. Brandt, J. M. Ford, National Radio Astronomy Observatory (United States); B. Kanevsky, Astro Space Ctr. of Lebedev Physical Institute (Russian Federation); A. Kovalenko, Pushchino Radio Astronomy Observatory, Astro Space Ctr. of Lebedev Physical Institute (Russian Federation);Y. Y. Kovalev, Astro Space Ctr. of Lebedev Physical Institute (Russian Federation); R. J. Maddalena, National Radio Astronomy Observatory (United States); S. Sergeev, Lavochkin Association (Russian Federation); A. Smirnov, Astro Space Ctr. of Lebedev Physical Institute (Russian Federation); G. Watts, T. L. Weadon, National Radio Astronomy Observatory (United States)

\section{SITE CHARACTERIZATION AND TESTING}

9145 OC Assessing VLT-UT science image quality from active optics Shack-Hartmann spot patterns [9145-11]

J. Navarrete, European Southern Observatory, LaSilla-Paranal Observatory (Chile); J. Kolb, European Southern Observatory (Germany); G. Lombardi, European Southern Observatory LaSilla-Paranal Observatory (Chile); L. Noethe, M. Sarazin, European Southern Observatory (Germany)

9145 OD Antarctic observations at long wavelengths with the IRAIT-ITM Telescope at Dome C [9145-12] G. A. Durand, Lab. AIM, CEA-IRFU, CNRS, Univ. Paris Diderot (France); P. Tremblin, Univ. of Exeter (United Kingdom); V. Minier, Lab. AIM, CEA-IRFU, CNRS, Univ. Paris Diderot (France); Y. Reinert, CEA-IRFU (France); C. Leroy dos Santos, Univ. degli Studi di Perugia (Italy); L. Rodriguez, X. Joffrin, Lab. AIM, CEA-IRFU, CNRS, Univ. Paris Diderot (France); M. Busso, G. Tosti, G. Nucciarelli, Univ. degli Studi di Perugia (Italy); M. Dolci, O. Straniero, A. Valentini, INAF - Osservatorio Astronomico di Teramo (Italy); C. Abia, Univ. de Granada (Spain); J. M. Christille, Osservatorio Astronomico della Regione Autonoma Valle d'Aosta (Italy); E. Doumayrou, M. Lortholary, Lab. AIM, CEA-IRFU, CNRS, Univ. Paris Diderot (France); P. Charron, P. Lotrus, C. Walter, CEA-IRFU (France); S. Ronayette, Lab. AIM, CEA-IRFU, CNRS, Univ. Paris Diderot (France); Z. Challita, Lab. d'Astrophysique de Marseille (France); L. Fromont, INRA - AgroParisTech (France); M. Condamin, Institut d'Astrophysique Spatiale Bâtiment 121, Univ. Paris XI (France); M. K. Kwon, Observatoire de Paris (France);

D. Tavagnacco, INAF - Osservatorio Astronomico di Tireste (Italy) 
9145 OE Kunlun Dark Universe Survey Telescope (Invited Paper) [9145-13]

Y. Zhu, National Astronomical Observatories, Nanjing Institute of Astronomical Optics \& Technology (China) and Key Lab. of Astronomical Optics \& Technology, Nanjing Institute of Astronomical Optics \& Technology (China); L. Wang, Purple Mountain Observatory (China) and Texas A\&M Univ. (United States); X. Yuan, B. Gu, X. Li, S. Yang, X. Gong, F. Du, Y. Qi, L. Xu, National Astronomical Observatories, Nanjing Institute of Astronomical Optics \& Technology (China) and Key Lab. of Astronomical Optics \& Technology, Nanjing Institute of Astronomical Optics \& Technology (China)

9145 OF The AST3 project: Antarctic Survey Telescopes for Dome A [9145-14]

X. Yuan, X. Cui, National Astronomical Observatories, Nanjing Institute of Astronomical Optics \& Technology (China) and Chinese Ctr. for Antarctic Astronomy (China); B. Gu, S. Yang, F. Du, X. Li, D. Wang, X. Li, National Astronomical Observatories, Nanjing Institute of Astronomical Optics \& Technology (China); X. Gong, National Astronomical Observatories, Nanjing Institute of Astronomical Optics \& Technology (China) and Chinese Ctr. for Antarctic Astronomy (China); H. Wen, Z. Li, H. LU, L. XU, R. Zhang, Y. Zhang, National Astronomical Observatories, Nanjing Institute of Astronomical Optics \& Technology (China); L. Wang, Chinese Ctr. for Antarctic Astronomy (China) and Purple Mountain Observatory (China); Z. Shang, Chinese Ctr. for Antarctic Astronomy (China), National Astronomical Observatories (China), and Tianjin Normal Univ. (China); Y. Hu, B. Ma, Q. Liu, P. Wei, National Astronomical Observatories (China)

9145 OG The Greenland Telescope (GLT): antenna status and future plans [9145-15] P. Raffin, J. C. Algaba-Marcosa, K. Asada, Institute of Astronomy and Astrophysics, Academia Sinica (Taiwan); R. Blundell, R. Burgos, Harvard-Smithsonian Ctr. for Astrophysics (United States); C.-C. Chang, M.-T. Chen, Institute of Astronomy and Astrophysics, Academia Sinica (Taiwan); R. Christensen, Smithsonian Astrophysical Observatory (United States); P. K. Grimes, Harvard-Smithsonian Ctr. for Astrophysics (United States); C. C. Han, Institute of Astronomy and Astrophysics, Academia Sinica (Taiwan); P. T. P. Ho, Institute of Astronomy and Astrophysics, Academia Sinica (Taiwan) and Harvard-Smithsonian Ctr. for Astrophysics (United States); Y.-D. Huang, M. Inove, P. M. Koch, Institute of Astronomy and Astrophysics, Academia Sinica (Taiwan); D. Kubo, Institute of Astronomy and Astrophysics, Academia Sinica (United States); S. Leiker, Harvard-Smithsonian Ctr. for Astrophysics (United States); C.-T. Liu, ASRD/CSIST (Taiwan); P. Martin-Cocher, S. Matsushita, M. Nakamura, H. Nishioka, G. Nystrom, Institute of Astronomy and Astrophysics, Academia Sinica (Taiwan); S. N. Paine, N. A. Patel, Harvard-Smithsonian Ctr. for Astrophysics (United States); N. Pradel, H.-Y. Pu, H.-Y. Shen, Institute of Astronomy and Astrophysics, Academia Sinica (Taiwan); W. Snow, Institute of Astronomy and Astrophysics, Academia Sinica (United States); T. K. Sridharan, Harvard-Smithsonian Ctr. for Astrophysics (United States); R. Srinivasan, Institute of Astronomy and Astrophysics, Academia Sinica (United States); E. Tong, Harvard-Smithsonian Ctr. for Astrophysics (United States); J. Wang, Institute of Astronomy and Astrophysics, Academia Sinica (Taiwan)

$9145 \mathrm{OH} \quad$ New results from the first exoplanet survey in the Canadian High Arctic [9145-16] N. M. Law, The Univ. of North Carolina at Chapel Hill (United States); R. Carlberg, Univ. of Toronto (Canada); O. Fors, The Univ. of North Carolina at Chapel Hill (United States); E. Steinbring, National Research Council Canada (Canada); W. Ngan, Univ. of Toronto (Canada); P. Wulfken, B. Pedersen, Univ. of North Carolina at Chapel Hill (United States); J. Maire, S. Sivanandam, Univ. of Toronto (Canada) 
9145 ol Ground-based astronomical instrument for planetary protection [9145-17]

R. L. Kendrick, D. Bennett, M. Bold, Lockheed Martin STAR Labs. (United States)

9145 0J Space Object Tracking (SPOT) facility [9145-18]

R. Shivitz, R. Kendrick, J. Mason, M. Bold, T. Kubo, K. Bock, D. Tyler, Lockheed Martin Space Systems Co. (United States)

\section{CHERENKOV TELESCOPES I}

9145 OK Status of the Cherenkov Telescope Array Project (Invited Paper) [9145-19]

S. Schlenstedt, Deutsches Elektronen-Synchrotron (Germany)

$9145 \mathrm{OL}$ The ASTRI SST-2M prototype for the Cherenkov Telescope Array: prototype technologies goals and strategies for the future SST [9145-20]

G. Marchiori, A. Busatta, S. Giacomel, European Industrial Engineering s.r.l. (Italy); I. Folla, M. Valsecchi, Galbiati Group Srl (Italy); R. Canestrari, G. Bonnoli, INAF - Osservatorio Astronomico di Brera (Italy); E. Cascone, INAF - Osservatorio Astronomico di Capodimonte (Italy); P. Conconi, INAF - Osservatorio Astronomico di Brera (Italy); M. Fiorini, INAF - IASF Milano (Italy); E. Giro, INAF - Osservatorio Astronomico di Padova (Italy); N. La Palombara, INAF - IASF Milano (Italy); G. Pareschi, L. Perri, INAF - Osservatorio Astronomico di Brera (Italy); G. Rodeghiero, INAF - Osservatorio Astronomico di Padova (Italy); G. Sironi, INAF Osservatorio Astronomico di Brera (Italy); L. Stringhetti, G. Toso, INAF - IASF Milano (Italy); G. Tosti, Univ. di Perugia (Italy); C. Pellicciari, INAF - Osservatorio Astronomico di Brera (Italy)

9145 OM The ASTRI SST-2M prototype for the Cherenkov Telescope Array: manufacturing of the structure and the mirrors [9145-21]

R. Canestrari, G. Bonnoli, INAF - Osservatorio Astronomico di Brera (Italy); E. Cascone, INAFOsservatorio Astronomico di Capodimonte (Italy); P. Conconi, G. Crimi, INAF - Osservatorio Astronomico di Brera (Italy); M. Fiorini, INAF - IASF Milano (Italy); E. Giro, INAF - Osservatorio Astronomico di Padova (Italy); N. La Palombara, INAF - IASF Milano (Italy); G. Pareschi, L. Perri, INAF - Osservatorio Astronomico di Brera (Italy); G. Rodeghiero, INAF - Osservatorio Astronomico di Padova (Italy); G. Sironi, INAF - Osservatorio Astronomico di Brera (Italy); L. Stringhetti, G. Toso, INAF - IASF Milano (Italy); G. Tosti, Univ. degli Studi di Perugia (Italy); C. Pelliciari, INAF - Osservatorio Astronomico di Brera (Italy)

\section{CHERENKOV TELESCOPES II}

914500 Medium-sized telescopes for the Cherenkov Telescope Array [9145-23]

S. Schlenstedt, Deutsches Elektronen-Synchrotron (Germany)

9145 OP The large size telescope of the Cherenkov Telescope Array [9145-24]

G. Ambrosi, INFN (Italy); Y. Awane, Kyoto Univ. (Japan); H. Baba, Ibaraki Univ. (Japan); A. Bamba, Aoyama Gakuin Univ. (Japan); M. Barceló, Institut de Física d'Altes Energies (Spain); U. Barres de Almeida, Ctr. Brasileiro de Pesquisas Fisicas (Brazil); J. A. Barrio, Univ. Complutense de Madrid (Spain); O. Blanch Bigas, J. Boix, Institut de Física d'Altes Energies (Spain); L. Brunetti, Lab. d'Annecy-le-Vieux de Physique des Particules, CNRS, Univ. de 
Savoie (France); E. Carmona, Ctr. de Investigaciones Energéticas, Medioambientales y Tecnológicas (Spain); E. Chabanne, Lab. d'Annecy-le-Vieux de Physique des Particules, CNRS, Univ. de Savoie (France); M. Chikawa, Kinki Univ. (Japan); R. Colin, Max-PlanckInstitut für Physik (Germany); J. Cortina, Institut de Física d'Altes Energies (Spain); J. L. Contreras, Univ. Complutense de Madrid (Spain); F. Dazzi, Istituto Nazionale di Fisica Nucleare (Italy); A. De Angelis, Univ. degli Studi di Udine (Italy) and Istituto Nazionale di Fisica Nucleare (Italy); G. Deleglise, Lab. d'Annecy-le-Vieux de Physique des Particules, CNRS, Univ. de Savoie (France); C. Delgado, C. Díaz, Ctr. de Investigaciones Energéticas, Medioambientales y Tecnológicas (Mexico); A. Fiasson, Lab. d'Annecy-le-Vieux de Physique des Particules, CNRS, Univ. de Savoie (France); D. Fink, Max-Planck-Institut für Physik (Germany); N. Fouque, Lab. d'Annecy-le-Vieux de Physique des Particules, CNRS, Univ. de Savoie (France); L. Freixas, Ctr. de Investigaciones Energéticas, Medioambientales y Tecnológicas (Spain); C. Fruck, Max-Planck-Institut für Physik (Germany); A. Gadola, Univ. of Zürich (Switzerland); R. García, Instituto de Astrofísica de Canarias (Spain); D. Gascon, Univ. de Barcelona (Spain); N. Geffroy, Lab. d'Annecy-le-Vieux de Physique des Particules, CNRS, Univ. de Savoie (France); N. Giglietto, F. Giordano, Istituto Nazionale di Fisica Nucleare (Italy); F. Grañena, Institut de Física d'Altes Energies (Spain); S. Gunji, Yamagata Univ. (Japan); R. Hagiwara, Yamagata Univ. (Japan); N. Hamer, Ctr. de Investigaciones Energéticas, Medioambientales y Tecnológicas (Spain); Y. Hanabata, The Univ. of Tokyo (Japan);. T. Hassan, Univ. Complutense de Madrid (Spain); K. Hatanaka, Kyoto Univ. (Japan); T. Haubold, Max-Planck-Institut für Physik (Germany); M. Hayashida, The Univ. of Tokyo (Japan); R. Hermel, Lab. d'Annecy-le-Vieux de Physique des Particules, CNRS, Univ. de Savoie (France); D. Herranz, Univ. Complutense de Madrid (Spain); K. Hirotani, S. Inove, Y. Inove, The Univ. of Tokyo (Japan); K. Ioka, Institute of Particle and Nuclear Studies, KEK (Japan); C. Jablonski, Max-Planck-Institut für Physik (Germany); M. Kagaya, H. Katagiri, Ibaraki Univ. (Japan); T. Kishimoto, Kyoto Univ. (Japan); K. Kodani, Tokai Univ. (Japan); K. Kohri, Institute of Particle and Nuclear Studies, KEK (Japan); Y. Konno, Kyoto Univ. (Japan); S. Koyama, Saitama Univ. (Japan); H. Kubo, Kyoto Univ. (Japan); J. Kushida, Tokai Univ. (Japan); G. Lamanna, T. Le Flour, Lab. d'Annecy-le-Vieux de Physique des Particules, CNRS, Univ. de Savoie (France); E. Lorenz, Max-Planck-Institut für Physik (Germany); R. López, Institut de Física d'Altes Energies (Spain); M. López-Moya, Univ. Complutense de Madrid (Spain); P. Majumdar, Saha Institute of Nuclear Physics (India); A. Manalaysay, Univ. of Zürich (Switzerland); M. Mariotti, Univ. degli Studi di Padova (Italy); G. Martínez, Ctr. de Investigaciones Energéticas, Medioambientales y Tecnológicas (Spain); M. Martínez, Institut de Física d'Altes Energies (Mexico); D. Mazin, Max-Planck-Institut für Physik (Germany); J. M. Miranda , Univ. Complutense de Madrid (Spain); R. Mirzoyan, MaxPlanck-Institut für Physik (Germany); I. Monteiro, Lab. d'Annecy-le-Vieux de Physique des Particules, CNRS, Univ. de Savoie (France); A. Moralejo, Institut de Física d'Altes Energies (Spain); K. Murase, The Univ. of Tokyo (Japan); S. Nagataki, RIKEN (Japan); D. Nakajima, Max-Planck-Institut für Physik (Germany); T. Nakamori, Waseda Univ. (Japan); K. Nishijima, Tokai Univ. (Japan); K. Noda, Max-Planck-Institut für Physik (Germany); A. Nozato, Kinki Univ. (Japan); Y. Ohira, Institute of Particle and Nuclear Studies (Japan); M. Ohishi, H. Ohoka, The Univ. of Tokyo (Japan); A. Okumura, Nagoya Univ. (Japan); R. Orito, Univ. of Tokushima (Japan); J. L. Panazol, Lab. d'Annecy-le-Vieux de Physique des Particules, CNRS, Univ. de Savoie (France); D. Paneque, Max-Planck-Institut für Physik (Germany); R. Paoletti, Univ. degli Studi di Siena (Italy) and Istituto Nazionale di Fisica Nucleare (Italy); J. M. Paredes, Univ. de Barcelona (Spain); G. Pauletta, Univ. degli Studi di Udine (Italy) and Istituto Nazionale di Fisica Nucleare (Italy); S. Podkladkin, Max-Planck-Institut für Physik (Germany); J. Prast, Lab. d'Annecy-le-Vieux de Physique des Particules, CNRS, Univ. de Savoie (France); R. Rando, Univ. degli Studi di Padova (Italy); O. Reimann, Max-PlanckInstitut für Physik (Germany); M. Ribó, Univ. de Barcelona (Spain); S. Rosier-Lees, Lab. d'Annecy-le-Vieux de Physique des Particules, CNRS, Univ. de Savoie (France); K. Saito, The 
Univ. of Tokyo (Japan); T. Saito, Kyoto Univ. (Japan); Y. Saito, Tokai Univ. (Japan); N. Sakaki, The Univ. of Tokyo (Japan); R. Sakonaka, Kinki Univ. (Japan); A. Sanuy, Univ. de Barcelona (Spain); H. Sasaki, Konan Univ. (Japan); M. Sawada, Aoyama Gakuin Univ. (Japan); V. Scalzotto, S. Schultz, Univ. degli Studi di Padova (Italy); T. Schweizer, Max-Planck-Institut für Physik (Germany); T. Shibata, Aoyama Gakuin Univ. (Japan); S. Shu, Kinki Univ. (Japan); J. Sieiro, Univ. de Barcelona (Spain); V. Stamatescu, Institut de Física d'Altes Energies (Spain); S. Steiner, U. Straumann, Univ. of Zürich (Switzerland); R. Sugawara, Univ. of Tokushima (Japan); H. Tajima, Nagoya Univ. (Japan); H. Takami, Institute of Particle and Nuclear Studies, KEK (Japan); S. Tanaka, Ibaraki Univ. (Japan); M. Tanaka, Institute of Particle and Nuclear Studies (Japan); L. A. Tejedor, Univ. Complutense de Madrid (Spain); Y. Terada, Saitama Univ. (Japan); M. Teshima, The Univ. of Tokyo (Japan) and Max-PlanckInstitut für Physik (Germany); T. Totani, The Univ. of Tokyo (Japan); H. Ueno, Saitama Univ. (Japan); K. Umehara, Ibaraki Univ. (Japan); A. Vollhardt, Univ. of Zürich (Switzerland); R. Wagner, H. Wetteskind, Max-Planck-Institut für Physik (Germany); T. Yamamoto, Konan Univ. (Japan); R. Yamazaki, A. Yoshida, Aoyama Gakuin Univ. (Japan); T. Yoshida, Ibaraki Univ. (Japan); T. Yoshikoshi, The Univ. of Tokyo (Japan)

\section{AIRBORNE TELESCOPES I}

$91450 Q \quad$ SOFIA general investigator science program (Invited Paper) [9145-25]

E. T. Young, B.-G. Andersson, E. E. Becklin, W. T. Reach, R. Sankrit, NASA Ames Research Ctr. (United States); H. Zinnecker, NASA Ames Research Ctr. (United States) and Univ. Stuttgart (Germany); A. Krabbe, Univ. Stuttgart (Germany)

9145 OR The Balloon-borne Large Aperture Submillimeter Telescope for Polarimetry-BLASTPol: performance and results from the 2012 Antarctic flight [9145-26]

N. Galitzki, Univ. of Pennsylvania (United States); P. A. R. Ade, Cardiff Univ. (United Kingdom); F. E. Angilè, Univ. of Pennsylvania (United States); S. J. Benton, Univ. of Toronto (Canada); M. J. Devlin, B. Dober, Univ. of Pennsylvania (United States); L. M. Fissel, Univ. of Toronto (Canada) and Northwestern Univ. (United States); Y. Fukui, Nagoya Univ. (Japan); N. N. Gandilo, Univ. of Toronto (Canada); J. Klein, Univ. of Pennsylvania (United States); A. L. Korotkov, Brown Univ. (United States); T. G. Matthews, Northwestern Univ. (United States); L. Moncelsi, California Institute of Technology (United States); C. B. Netterfield, Univ. of Toronto (Canada) and Canadian Institute for Advanced Research (Canada); G. Novak, Northwestern Univ. (United States); D. Nutter, E. Pascale, Cardiff Univ. (United Kingdom); F. Poidevin, Instituto de Astrofísica de Canarias (Spain) and Univ. de La Laguna (Spain); G. Savini, Univ. College London (United Kingdom); D. Scott, Northwestern Univ. (United States); J. A. Shariff, Univ. of Toronto (Canada); J. D. Soler, Univ. of Toronto (Canada) and Institut d'Astrophysique Spatiale (France); C. E. Tucker, Cardiff Univ. (United Kingdom); G. S. Tucker, Brown Univ. (United States); D. Ward-Thompson, Univ. of Central Lancashire (United Kingdom)

9145 OS SOFIA pointing history [9145-27]

H. J. Kärcher, MT Mechatronics GmbH (Germany); N. Kunz, P. Temi, NASA Ames Research Ctr. (United States); A. Krabbe, J. Wagner, Deutsches SOFIA Institut, Univ. Stuttgart

(Germany); M. Süß, MT Mechatronics GmbH (Germany) 
9145 OT Design and construction of a carbon fiber gondola for the SPIDER balloon-borne telescope [9145-28]

J. D. Soler, Institut d'Astrophysique Spatiale, CNRS (France) and Univ. of Toronto (Canada); P. A. R. Ade, Cardiff Univ. (United Kingdom); M. Amiri, The Univ. of British Columbia (Canada); S. J. Benton, Univ. of Toronto (Canada); J. J. Bock, California Institute of Technology (United States); J. R. Bond, Canadian Institute for Theoretical Astrophysics, Univ. of Toronto (Canada) and Canadian Institute for Advanced Research (Canada); S. A. Bryan, Case Western Reserve Univ. (United States); C. Chiang, Princeton Univ. (United States) and Univ. of KwaZulu-Natal (South Africa); C. C. Contaldi, Imperial College London (United Kingdom); B. P. Crill, O. P. Doré, California Institute of Technology (United States) and Jet Propulsion Lab. (United States); M. Farhang, Univ. of Toronto (Canada); J. P. Filippini, California Institute of Technology (United States); L. M. Fissel, Univ. of Toronto (Canada); A. A. Fraisse, A. E. Gambrel, Princeton Univ. (United States); N. N. Gandilo, Univ. of Toronto (Canada); S. Golwala, California Institute of Technology (United States); J. E. Gudmundsson, Princeton Univ. (United States); M. Halpern, The Univ. of British Columbia (Canada) and Canadian Institute for Advanced Research (Canada); M. Hasselfield, The Univ. of British Columbia (Canada) and Princeton Univ. (United States); G. C. Hilton, National Institute of Standards and Technology (United States); W. A. Holmes, Jet Propulsion Lab. (United States); V. V. Hristov, California Institute of Technology (United States); K. D. Irwin, National Institute of Standards and Technology (United States); W. C. Jones, Z. K. Kermish, Princeton Univ. (United States); C.-L. Kuo, Stanford Univ. (United States); C. J. MacTavish, Canadian Institute for Theoretical Astrophysics, Univ. of Toronto (Canada); P. V. Mason, California Institute of Technology (United States); K. G. Megerian, Jet Propulsion Lab. (United States); L. Moncelsi, T. Morford, California Institute of Technology (United States); J. M. Nagy, Case Western Reserve Univ. (United States); C. B. Netterfield, Univ. of Toronto (Canada) and Canadian Institute for Advanced Research (Canada); A. S. Rahlin, Princeton Univ. (United States); C. D. Reintsema, National Institute of Standards and Technology (United States); J. E. Ruhl, Case Western Reserve Univ. (United States); M. C. Runyan, Jet Propulsion Lab. (United States); J. A. Shariff, Univ. of Toronto (Canada); A. Trangsrud, Jet Propulsion Lab. (United States); C. Tucker, Cardiff Univ. (United Kingdom); R. S. Tucker, California Institute of Technology (United States); A. D. Turner, A. C. Weber, Jet Propulsion Lab. (United States); D. V. Wiebe, The Univ. of British Columbia (Canada); E. Y. Young, Princeton Univ. (United States)

9145 OU Pointing control for the SPIDER balloon-borne telescope [9145-102] J. A. Shariff, Univ. of Toronto (Canada); P. A. R. Ade, Cardiff Univ. (United Kingdom); M. Amiri, The Univ. of British Columbia (Canada); S. J. Benton, Univ. of Toronto (Canada); J. J. Bock, California Institute of Technology (United States) and Jet Propulsion Lab. (United States); J. R. Bond, Canadian Institute for Theoretical Astrophysics, Univ. of Toronto (Canada) and Canadian Institute for Advanced Research (Canada); S. A. Bryan, Case Western Reserve Univ. (United States); H. C. Chiang, Univ. of KwaZulu-Natal (South Africa); C. R. Contaldi, Imperial College London (United Kingdom); B. P. Crill, O. P. Doré, California Institute of Technology (United States) and Jet Propulsion Lab. (United States); M. Farhang, Univ. of Toronto (Canada) and Canadian Institute for Theoretical Astrophysics, Univ. of Toronto (Canada); J. P. Filippini, California Institute of Technology (United States); L. M. Fissel, Univ. of Toronto (Canada) and Northwestern Univ. (Canada); A. A. Fraisse, A. E. Gambrel, Princeton Univ. (United States); N. N. Gandilo, Univ. of Toronto (Canada); S. R. Golwala, California Institute of Technology (United States); J. E. Gudmundsson, Princeton Univ. (United States); M. Halpern, The Univ. of British Columbia (Canada) and 
Canadian Institute for Advanced Research (Canada); M. Hasselfield, Princeton Univ. (United States); G. C. Hilton, National Institute of Standards and Technology (United States); W. A. Holmes, Jet Propulsion Lab. (United States); V. V. Hristov, California Institute of Technology (United States); K. D. Irwin, National Institute of Standards and Technology (United States), Stanford Univ. (United States), and SLAC National Accelerator Lab., Kavli Institute for Particle Astrophysics and Cosmology (United States); W. C. Jones, Z. D. Kermish, Princeton Univ. (United States); J. Klein, Univ. of Pennsylvania (United States); A. L. Korotov, Brown Univ. (United States); C. L. Kuo, Stanford Univ. (United States) and SLAC National Accelerator Lab., Kavli Institute for Particle Astrophysics and Cosmology (United States); C. J. MacTavish, Kavli Institute for Cosmology, Univ. of Cambridge (United Kingdom); P. V. Mason, California Institute of Technology (United States); K. G. Megerian, Jet Propulsion Lab. (United States); L. Moncelsi, T. A. Morford, California Institute of Technology (United States); J. M. Nagy, Case Western Reserve Univ. (United States); C. B. Netterfield, Univ. of Toronto (Canada) and Canadian Institute for Advanced Research (Canada); R. O'Brient, California Institute of Technology (United States) and Jet Propulsion Lab. (United States); A. S. Rahlin, Princeton Univ. (United States); C. D. Reintsema, National Institute of Standards and Technology (United States); J. E. Ruhl, Case Western Reserve Univ. (United States); M. C. Runyan, Jet Propulsion Lab. (United States); J. D. Soler, Univ. of Toronto (France) and Institut d'Astrophysique Spatiale, CNRS, Univ. Paris-Sud (France); A. Trangsrud, Jet Propulsion Lab. (United States); C. E. Tucker, Cardiff Univ. (United Kingdom); R. S. Tucker, California Institute of Technology (United States); A. D. Turner, A. C. Weber, Jet Propulsion Lab. (United States); D. V. Wiebe, The Univ. of British Columbia (Canada); E. Y. Young, Princeton Univ. (United States)

9145 OV BLASTbus electronics: general-purpose readout and control for balloon-borne experiments [9145-30]

S. J. Benton, Univ. of Toronto (Canada); P. A. Ade, Cardiff Univ. (United Kingdom); M. Amiri, The Univ. of British Columbia (Canada); F. E. Angilè, Univ. of Pennsylvania (United States); J. J. Bock, California Institute of Technology (United States) and Jet Propulsion Lab. (United States); J. R. Bond, Canadian Institute for Theoretical Astrophysics (Canada) and Canadian Institute for Advanced Research (Canada); S. A. Bryan, Case Western Reserve Univ. (United States); H. C. Chiang, Univ. of KwaZulu-Natal (South Africa); C. R. Contaldi, Imperial College London (United Kingdom); B. P. Crill, California Institute of Technology (United States) and Jet Propulsion Lab. (United States); M. J. Devlin, B. Dober, Univ. of Pennsylvania (United States); O. P. Doré, California Institute of Technology (United States) and Jet Propulsion Lab. (United States); C. D. Dowell, Jet Propulsion Lab. (United States); M. Farhang, Canadian Institute for Theoretical Astrophysics (Canada) and Univ. of Toronto (Canada); J. P. Filippini, California Institute of Technology (United States); L. M. Fissel, Northwestern Univ. (United States) and Univ. of Toronto (Canada); A. A. Fraisse, Princeton Univ. (United States); Y. Fukui, Nagoya Univ. (Japan); N. Galitzki, Univ. of Pennsylvania (United States); A. E. Gambrel, Princeton Univ. (United States); N. N. Gandilo, Univ. of Toronto (Canada); S. R. Golwala, California Institute of Technology (United States); J. E. Gudmundsson, Princeton Univ. (United States); M. Halpern, The Univ. of British Columbia (Canada) and Canadian Institute for Advanced Research (Canada); M. Hasselfield, Princeton Univ. (Canada); G. C. Hilton, National Institute of Standards and Technology (United States); W. A. Holmes, Jet Propulsion Lab. (United States); V. V. Hristov, California Institute of Technology (United States); K. D. Irwin, Stanford Univ. (United States), SLAC National Accelerator Lab., Kavli Institute for Particle Astrophysics and Cosmology (United States), and National Institute of Standards and Technology (United States); W. C. Jones, Z. D. Kermish, Princeton Univ. (United States); J. Klein, Univ. of Pennsylvania (United States); A. L. Korotkov, Brown Univ. (United States); C. L. Kuo, Stanford Univ. (United States) and SLAC National Accelerator Lab., Kavli Institute for Particle Astrophysics and 
Cosmology (United States); C. J. MacTavish, Kavli Institute for Cosmology, Univ. of Cambridge (United Kingdom); P. V. Mason, California Institute of Technology (United States); T. G. Matthews, Northwestern Univ. (United States); K. G. Megerian, Jet Propulsion Lab. (United States); L. Moncelsi, T. A. Morford, T. K. Mroczkowski, California Institute of Technology (United States); J. M. Nagy, Case Western Reserve Univ. (United States); C. B. Netterfield, Univ. of Toronto (Canada) and Canadian Institute for Advanced Research (Canada); G. Novak, Northwestern Univ. (United States); D. Nutter, Cardiff Univ. (United Kingdom); R. O'Brient, California Institute of Technology (United States) and Jet Propulsion Lab. (United States); R. W. Ogburn, IV, Kavli Institute for Particle Astrophysics and Cosmology, SLAC National Accelerator Lab. (United States); E. Pascale, Cardiff Univ. (United Kingdom); F. Poidevin, Instituto de Astrofísica de Canarias (Spain) and Univ. de La Laguna (Spain); A. S. Rahlin, Princeton Univ. (United States); C. D. Reintsema, National Institute of Standards and Technology (United States); J. E. Ruhl, Case Western Reserve Univ. (United States); M. C. Runyan, Jet Propulsion Lab. (United States); G. Savini, Univ. College London (United Kingdom); D. Scott, The Univ. of British Columbia (Canada); J. A. Shariff, Univ. of Toronto (Canada); J. D. Soler, Institut d'Astrophysique Spatiale, CNRS, Univ. Paris-Sud (France) and Univ. of Toronto (Canada); N. E. Thomas, Univ. of Miami (United States); A. Trangsrud, Jet Propulsion Lab. (United States); M. D. Truch, Univ. of Pennsylvania (United States); C. E. Tucker, Cardiff Univ. (United Kingdom); G. S. Tucker, Brown Univ. (United States); R. S. Tucker, California Institute of Technology (United States); A. D. Turner, Jet Propulsion Lab. (United States); D. Ward-Thompson, Univ. of Toronto (Canada) and Cardiff Univ. (United Kingdom); A. C. Weber, Jet Propulsion Lab. (United States); D. V. Wiebe, The Univ. of British Columbia (Canada); E. Y. Young, Princeton Univ. (United States)

9145 OW Upgrade of the SOFIA target acquisition and tracking cameras [9145-31] J. Wolf, M. Wiedemann, E. Pfüller, M. Lachenmann, Deutsches SOFIA Institut, Univ. Stuttgart (Germany) and SOFIA Science Ctr., NASA Ames Research Ctr. (United States); H. J. Hall, SOFIA/USRA (United States); H.-P. Röser, Deutsches SOFIA Institut, Univ. Stuttgart (Germany)

TELESCOPES AND ARRAYS FOR SURVEYS, TIME-DOMAIN AND TRANSIENT OBSERVATIONS I

$91450 Y \quad$ The Pan-STARRS Project in 2014 (Invited Paper) [9145-33]

J. S. Morgan, W. Burgett, P. Onaka, Institute for Astronomy, Univ. of Hawai'i (United States)

$9145 \mathrm{OZ}$ The Evryscope: the first full-sky gigapixel-scale telescope [9145-144]

N. M. Law, O. Fors, P. Wulfken, J. Ratzloff, D. Kavanaugh, The Univ. of North Carolina at Chapel Hill (United States)

914510 Introduction of Chinese SONG telescope [9145-35]

G. Wang, X. Jiang, H. Wang, S. Kou, D. Niu, Y. Ye, Z. Zhang, J. Xu, C. Ren, L. Xu, Z. Yue, National Astronomical Observatories, Nanjing Institute of Astronomical Optics \& Technology (China) and Key Lab. of Astronomical Optics \& Technology, Nanjing Institute of Astronomical Optics \& Technology (China)

914511 Liverpool Telescope 2: a new robotic facility for time domain astronomy in 2020+ [9145-36] C. M. Copperwheat, I. A. Steele, S. D. Bates, R. J. Smith, M. F. Bode, Liverpool John Moores Univ. (United Kingdom); I. Baker, Glyndwr Innovations Ltd. (United Kingdom); T. Peacocke, Lyncaeus Ltd. (United Kingdom); K. Thomson, Glyndwr Innovations Ltd. (United Kingdom) 
914513 Status of the Transneptunian Automated Occultation Survey (TAOS II) [9145-38]

M. J. Lehner, Institute of Astronomy and Astrophysics, Academia Sinica (Taiwan), Univ. of Pennsylvania (United States), and Harvard-Smithsonian Ctr. for Astrophysics (United States); S.-Y. Wang, Institute of Astronomy and Astrophysics, Academia Sinica (Taiwan); C. A. Alcock, Harvard-Smithsonian Ctr. for Astrophysics (United States); K. H. Cook, Institute of Astronomy and Astrophysics, Academia Sinica (Taiwan); G. Furesz, J. C. Geary, HarvardSmithsonian Ctr. for Astrophysics (United States); D. Hiriart, Instituto de Astronomía, Univ. Nacional Autónoma de México (Mexico); P. T. Ho, Institute of Astronomy and Astrophysics, Academia Sinica (Taiwan); W. H. Lee, Univ. Nacional Autónoma de México (Mexico); F. Melsheimer, DFM Engineering, Inc. (United States); T. Norton, Harvard-Smithsonian Ctr. for Astrophysics (United States); M. Reyes-Ruiz, M. Richer, Instituto de Astronomía, Univ. Nacional Autónoma de México (Mexico); A. Szentgyorgyi, Harvard-Smithsonian Ctr. for Astrophysics (United States); W.-L. Yen, Z.-W. Zhang, Institute of Astronomy and Astrophysics, Academia Sinica (Taiwan)

914514 MASCARA: the multi-site all-sky CAameRA: concept and first results [9145-39] A.-L. Lesage, J. F. P. Spronck, R. Stuik, Leiden Observatory, Leiden Univ. (Netherlands); F. Bettonvil, Leiden Observatory, Leiden Univ. (Netherlands) and NOVA Optical-Infrared Instrumentation Group at ASTRON (Netherlands); D. Pollaco, The Univ. of Warwick (United Kingdom); I. A. G. Snellen, Leiden Observatory, Leiden Univ. (Netherlands)

914515 Current status and future plans for the Maunakea Spectroscopic Explorer (MSE) [9145-40] D. A. Simons, Canada-France-Hawaii Telescope Corp. (United States); D. Crampton, P. Côté, A. McConnachie, K. Szeto, National Research Council- Herzberg (Canada); D. Salmon, D. Devost, Canada-France-Hawaii Telescope Corp. (United States); R. Murowinski, National Research Council - Herzberg (Canada)

914516 Wavefront sensing and the active optics system of the dark energy camera [9145-41] A. Roodman, K. Reil, C. Davis, SLAC National Accelerator Lab., Kavli Institute for Particle Astrophysics and Cosmology, Stanford Univ. (United States)

TELESCOPES AND ARRAYS FOR SURVEYS, TIME-DOMAIN AND TRANSIENT OBSERVATIONS III

914517 Spectroscopic survey of LAMOST (Invited Paper) [9145-42]

Y. Zhao, National Astronomical Observatories (China)

914518 Baseline design of the LSST telescope mount assembly [9145-43]

D. R. Neill, E. Hileman, J. Sebag, W. Gressler, O. Wiecha, M. Warner, J. Andrew, B. Schoening, National Optical Astronomy Observatory (United States)

914519 The Maunakea Spectroscopic Explorer: the science-driven design rationale [9145-44] A. McConnachie, R. Murowinski, D. Salmon, D. Simons, Canada-France-Hawaii Telescope (United States); P. Côté, NRC - Herzberg Institute of Astrophysics (Canada)

$91451 \mathrm{~A} \quad$ LSST Telescope and site status [9145-45]

W. Gressler, J. DeVries, E. Hileman, D. R. Neill, J. Sebag, O. Wiecha, J. Andrew, P. Lotz, W. Schoening, National Optical Astronomy Observatory (United States) 
9145 1B Real time estimation of differential piston at the LBT [9145-165]

M. Böhm, Univ. Stuttgart (Germany) and Max-Planck-Institut für Astronomie (Germany);

J.-U. Pott, Max-Planck-Institut für Astronomie (Germany); O. Sawodny, Univ. Stuttgart

(Germany); T. Herbst, M. Kürster, Max-Planck-Institut für Astronomie (Germany)

\section{EXTREMELY LARGE TELESCOPES I}

9145 1C Overview and status of the Giant Magellan Telescope project (Invited Paper) [9145-47]

R. A. Bernstein, P. J. McCarthy, The Giant Magellan Telescope Project Corp. (United States) and Carnegie Observatories (United States); K. Raybould, B. C. Bigelow, A. H. Bouchez, J. M. Filgueira, G. H. Jacoby, The Giant Magellan Telescope Project Corp. (United States); M. Johns, The Giant Magellan Telescope Project Corp. (United States) and Carnegie Observatories (United States); D. Sawyer, The Giant Magellan Telescope Project Corp. (United States); S. Shectman, Carnegie Observatories (United States); M. Sheehan, The Giant Magellan Telescope Project Corp. (United States)

9145 IE European Extremely Large Telescope: progress report (Invited Paper) [9145-49]

R. Tamai, J. Spyromilio, European Southern Observatory (Germany)

\section{EXTREMELY LARGE TELESCOPES II}

9145 IF Design of the Giant Magellan Telescope [9145-50]

M. Johns, C. Hull, G. Muller, B. Irarrazaval, A. Bouchez, T. Chylek, C. Smith, A. Wadhavkar, B. Bigelow, The Giant Magellan Telescope Project Corp. (United States); S. Gunnels, Paragon Engineering (United States); B. McLeod, Smithsonian Astrophysical Observatory (United States); C. Buleri, Quartus Engineering Inc. (United States)

9145 1G Looking beyond 30m-class telescopes: the Colossus project [9145-51] J. R. Kuhn, Institute of Astronomy, Univ. of Hawai'i (United States); S. V. Berdyugina, Kiepenhever-Institut für Sonnenphysik (Germany) and NASA Astrobiology Institute, Univ. of Hawai'i (United States); M. Langlois, G. Moretto, E. Thiébaut, Ctr. de Recherche Astrophysique de Lyon, CNRS, Observatoire de Lyon (France); C. Harlingten, D. Halliday, Innovative Optics Ltd. (Canada)

\section{ENABLING TECHNOLOGIES FOR EXTREMELY LARGE TELESCOPES I}

$91451 \mathrm{H} \quad$ GMT primary mirror support [9145-52]

C. Hull, The Giant Magellan Telescope Project Corp. (United States)

914511 The secondary mirror concept for the European Extremely Large Telescope [9145-53] M. Mueller, M. Cayrel, H. Bonnet, E. Ciattaglia, M. Esselborn, F. Koch, H. Kurlandczyk, L. Pettazzi, A. Rakich, B. Sedghi, European Southern Observatory (Germany) 
9145 1J High volume production trial of mirror segments for the Thirty Meter Telescope [9145-54] T. Oota, M. Negishi, H. Shinonaga, A. Gomi, Y. Tanaka, K. Akutsu, I. Otsuka, S. Mochizuki, Canon Inc. (Japan); M. Iye, T. Yamashita, National Astronomical Observatory of Japan (Japan)

\section{ENABLING TECHNOLOGIES FOR EXTREMELY LARGE TELESCOPES II}

$91451 \mathrm{~K}$ Improved E-ELT subsystem and component specifications, thanks to $\mathrm{M} 1$ test facility [9145-55]

M. Dimmler, J. Marrero, S. Leveque, P. Barriga, B. Sedghi, N. Kornweibel, European Southern Observatory (Germany)

9145 1L New strategies for an extremely large telescope dedicated to extremely high contrast: the Colossus project [9145-56]

G. Moretto, Ctr. de Recherche Astrophysique de Lyon, CNRS, Observatoire de Lyon (France); J. R. Kuhn, Institute for Astronomy, Univ. of Hawai'i (United States); E. Thiébaut, M. Langlois, Ctr. de Recherche Astrophysique de Lyon, CNRS, Observatoire de Lyon (France); S. V. Berdyugina, Kiepenheuer-Institut für Sonnenphysik (Germany) and NASA Astrobiology Institute, Univ. of Hawai'i (United States); C. Harlingten, D. Halliday, Innovative Optics Ltd. (Canada)

9145 1M Development of GMT fast steering secondary mirror assembly [9145-57]

M. Cho, National Optical Astronomy Observatory (United States); A. Corredor, C. Dribusch, W. H. Park, The Univ. of Arizona (United States); G. Muller, M. Johns, C. Hull, J. Kern, Giant Magellan Telescope Project (United States); Y.-S. Kim, Giant Magellan Telescope Project (United States) and Korea Astronomy and Space Science Institute (Korea, Republic of)

914510 Status of E-ELT M5 scale-one demonstrator [9145-59]

P. Barriga, B. Sedghi, M. Dimmler, N. Kornweibel, European Southern Observatory (Germany)

\section{Part Two}

\section{TELESCOPE CONTROLS}

9145 IP CCAT mount control using de-convolution for fast scans [9145-60]

P. M. Thompson, Systems Technology, Inc. (United States); S. Padin, California Institute of Technology (United States)

\section{SEGMENTED MIRROR ALIGNMENT, PHASING AND WAVEFRONT CONTROL}

$91451 Q$ On-sky measurement accuracy of Keck Telescope segment surface errors [9145-61] M. Troy, Jet Propulsion Lab. (United States); G. Chanan, Univ. of California, Irvine (United States); J. Roberts, Jet Propulsion Lab. (United States) 
9145 IR Inductive sensors based on embedded coil technology for nanometric inter-segment position sensing of the E-ELT [9145-62]

M. Wasmeier, J. Hackl, Micro-Epsilon Messtechnik GmbH \& Co. KG (Germany); S. Leveque, European Southern Observatory (Germany)

9145 is High performance control of mirror segment actuators for the European Extremely Large Telescope [9145-188]

G. Witvoet, R. den Breeje, J. Nijenhuis, R. Hazelebach, N. Doelman, TNO (Netherlands)

9145 1T The Giant Magellan Telescope active optics system [9145-64]

B. McLeod, Harvard-Smithsonian Ctr. for Astrophysics (United States); A. H. Bouchez,

B. Espeland, J. Filgueira, M. Johns, Giant Magellan Telescope (United States); T. J. Norton, M. Ordway, W. A. Podgorski, J. Roll, Harvard-Smithsonian Ctr. for Astrophysics (United States); C. Smith, Giant Magellan Telescope (United States)

$91451 \mathrm{U} \quad$ Fast optical re-phasing of segmented primary mirrors [9145-65]

H. Bonnet, M. Esselborn, N. Kornweibel, P. Dierickx, European Southern Observatory (Germany)

\section{MILLIMETER WAVELENGTH TELESCOPES AND ARRAYS I}

$91451 \mathrm{~V}$ Atacama large millimeter/submillimeter array: progress, status, and development (Invited Paper) [9145-66]

S. Corder, P. Cox, Joint ALMA Observatory (Chile)

$91451 \mathrm{~W} \quad$ Design of the optical system for ALMA band 1 [9145-67]

N. Reyes, Univ. de Chile (Chile) and Max-Planck-Institut für Radioastronomie (Germany); V. Tapia, Univ. de Chile (Chile); D. Henke, NRC - Herzburg Institute of Astrophysics (Canada); M. Sanchez-Carrasco, Univ. de Chile (Chile) and Instituto de Astrofísica de Andalucía (Spain); F. P. Mena, Univ. de Chile (Chile); S. M. X. Claude, NRC - Herzburg Institute of Astrophysics (Canada); L. Bronfman, Univ. de Chile (Chile)

$91451 Y \quad$ ALMA; the completion of the 25 Europeans antennas: focus on main performances, problem found during erection and lesson learned [9145-69] G. Marchiori, F. Rampini, L. Giacomel, S. Giacomel, E. Marcuzzi, F. Formentin, European Industrial Engineering s.r.l. (Italy)

\section{MILLIMETER WAVELENGTH TELESCOPES AND ARRAYS II}

914512 Development of high-accuracy pointing verification for ALMA antenna [9145-70]

A. Matsuzawa, The Graduate Univ. for Advanced Studies (Japan); M. Saito, The Graduate Univ. for Advanced Studies (Japan), Joint ALMA Observatory (Chile), and National Astronomical Observatory of Japan (Japan); S. Iguchi, The Graduate Univ. for Advanced Studies (Japan) and National Astronomical Observatory of Japan (Japan); K. Nakanishi, The Graduate Univ. for Advanced Studies (Japan), Joint ALMA Observatory (Chile), and National Astronomical Observatory of Japan (Japan); H. Saito, Nippon Institute of Technology (Japan) 
914520 What are scientifically valuable developments for ALMA enhancement? [9145-71]

S. Iguchi, D. Iono, National Astronomical Observatory of Japan (Japan) and The Graduate Univ. of Advanced Studies (Japan)

\section{RADIO TELESCOPES AND ARRAYS}

914522 Canadian Hydrogen Intensity Mapping Experiment (CHIME) Pathfinder [9145-73]

K. Bandura, McGill Univ. (Canada); G. E. Addison, M. Amiri, The Univ. of British Columbia (Canada); J. R. Bond, Canadian Institute for Theoretical Astrophysics (Canada) and Canadian Institute for Advanced Research (Canada); D. Campbell-Wilson, Univ. of Sydney (Australia); L. Connor, Canadian Institute for Theoretical Astrophysics (Canada) and Univ. of Toronto (Canada); J.-F. Cliche, McGill Univ. (Canada); G. Davis, M. Deng, The Univ. of British Columbia (Canada); N. Denman, Univ. of Toronto (Canada); M. Dobbs, McGill Univ. (Canada); M. Fandino, K. Gibbs, The Univ. of British Columbia (Canada); A. Gilbert, McGill Univ. (Canada); M. Halpern, The Univ. of British Columbia (Canada); D. Hanna, McGill Univ. (Canada); A. D. Hincks, G. Hinshaw, C. Höfer, The Univ. of British Columbia (Canada); P. Klages, Univ. of Toronto (Canada) and IBM Canada (Canada); T. L. Landecker, Dominion Radio Astrophysical Observatory, National Research Council Canada (Canada); K. Masui, The Univ. of British Columbia (Canada) and Canadian Institute for Advanced Research (Canada); J. Mena Parra, McGill Univ. (Canada); L. B. Newburgh, Univ. of Toronto (Canada); U.-L. Pen, Canadian Institute for Theoretical Astrophysics (Canada); J. B. Peterson, Carnegie Mellon Univ. (United States); A. Recnik, Univ. of Toronto (Canada); J. R. Shaw, Canadian Institute for Theoretical Astrophysics (Canada); K. Sigurdson, M. Sitwell, The Univ. of British Columbia (Canada); G. Smecher, McGill Univ. (Canada); R. Smegal, The Univ. of British Columbia (Canada); K. Vanderlinde, Univ. of Toronto (Canada); D. Wiebe, The Univ. of British Columbia (Canada)

914523 Using feed array networks to control distortions in antenna reflector for astrophysical radioastronomy [9145-74]

F. Centurelli, P. Monsurrò, Univ. degli Studi di Roma La Sapienza (Italy); F. Romano, Rheinmetall Italia SpA (Italy); G. Scotti, P. Tommasino, A. Trifeletti, Univ. degli Studi di Roma La Sapienza (Italy)

914524 A 200-GHz telescope unit for the QUIJOTE CMB Experiment [9145-75]

R. Sanquirce, B. Ełxeita, G. Murga, E. Fernandez, I. Sainz, IDOM Ingenieria y Consultoria S.A. (Spain); V. Sánchez, T. A. Viera-Curbelo, M. F. Gómez, M. Aguiar-Gonzalez, R. J. Hoyland, Á. R. Pérez de Taoro, A. Vega, R. Rebolo-López, J. A. Rubiño, Instituto de Astrofísica de Canarias (Spain)

SOLAR TELESCOPES I

914525 Construction status of the Daniel K. Inouye Solar Telescope (Invited Paper) [9145-76]

J. P. McMullin, National Solar Observatory (United States); T. R. Rimmele, V. Martínez Pillet, T. E. Berger, National Solar Observatory, Univ. of Colorado at Boulder (United States); R. Casini, NCAR High Altitude Observatory (United States); S. C. Craig, National Solar Observatory (United States); D. F. Elmore, National Solar Observatory, Univ. of Colorado at Boulder (United States); B. D. Goodrich, National Solar Observatory (United States); S. L. Hegwer, National Solar Observatory, Univ. of Colorado at Boulder (United States); R. P. Hubbard, E. M. Johansson, National Solar Observatory (United States); J. R. Kuhn, 
H. Lin, Institute for Astronomy, Univ. of Hawai'i (United States); W. McVeigh, National Solar Observatory (United States); W. Schmidt, Kiepenhever-Institut für Sonnenphysik (Germany); S. Shimko, A. Tritschler, M. Warner, National Solar Observatory (United States); F. Wöger, National Solar Observatory, Univ. of Colorado at Boulder (United States)

914526 The progress of Chinese Giant Solar Telescope [9145-77]

Z. Liu, Z. Jin, S. Yuan, J. Lin, Yunnan Astronomical Observatory (China); Y. Deng, National Astronomical Observatories (China); H. Ji, Purple Mountain Observatory (China); Y. Yan, National Astronomical Observatories (China)

914527 DKIST enclosure fabrication factory assembly and testing [9145-78]

G. Murga, AEC IDOM (United States); H. K. Marshall, National Solar Observatory (United States); T. E. Lorentz, J. Ariño, P. Ampuero, AEC IDOM (United States)

$914529 \quad$ 1.8-M solar telescope in China: the CLST [9145-80]

C. Rao, N. Gu, L. Zhu, Institute of Optics and Electronics (China) and The Key Lab. on Adaptive Optics (China); Y. Liu, Institute of Optics and Electronics (China), The Key Lab. on Adaptive Optics (China), and The Univ. of Chinese Academy of Sciences (China); J. Huang, Institute of Optics and Electronics (China); C. Li, Institute of Optics and Electronics (China) and The Key Lab. on Adaptive Optics (China); Y. Cheng, X. Cao, M. Zhang, Institute of Optics and Electronics (China); L. Zhang, Institute of Optics and Electronics (China), The Key Lab. on Adaptive Optics (China), and The Univ. of Chinese Academy of Sciences (China); H. Liu, Y. Wan, Institute of Optics and Electronics (China); $\mathrm{H}$. Xian, Institute of Optics and Electronics (China) and The Key Lab. on Adaptive Optics (China); W. Ma, Institute of Optics and Electronics (China); H. Bao, X. Zhang, C. Guan, D. Chen, M. Li, Institute of Optics and Electronics (China) and The Key Lab. on Adaptive Optics (China)

\section{SOLAR TELESCOPES II}

9145 2A Performance verification of the DKIST Mount and Coudé Laboratory [9145-81]

O. Dreyer, A. Ippa, S. Seubert, H. J. Kärcher, MT Mechatronics GmbH (Germany); P. Jeffers, National Solar Observatory (United States); G. Bonomi, Ingersoll Machines Tools, Inc. (United States)

\section{AIV, COMMISSIONING AND EARLY OPERATIONS}

$91452 \mathrm{~B}$ The automated planet finder at Lick Observatory [9145-85]

M. V. Radovan, K. Lanclos, B. P. Holden, R. I. Kibrick, S. L. Allen, W. T. S. Deich, E. Rivera, J. Burt, Univ. of California Observatories (United States); B. Fulton, Institute for Astronomy, Univ. of Hawaili (United States); P. Butler, Carnegie Institution of Washington (United States); S. S. Vogt, Univ. of California Observatories (United States) 
9145 2C Status and performance of the Discovery Channel Telescope from commissioning into early science operations [9145-82]

W. T. DeGroff, S. E. Levine, T. A. Bida, F. Cornelius, P. L. Collins, E. W. Dunham, B. Hardesty, M. Lacasse, M. Sweaton, Lowell Observatory (United States); A. J. Venetiou, L-3 Communications Integrated Optical Systems (United States); S. Zoonemat Kermani, P. Massey, M. L. Foley, H. Larson, J. Sanborn, S. Strosahl, R. Winner, T. Pugh, Lowell Observatory (United States)

9145 2D Commissioning and science verification of the $2 \mathrm{~m}$-Fraunhofer Wendelstein Telescope [9145-83]

U. Hopp, R. Bender, F. Grupp, Univ.-Sternwarte München (Germany) and Max-PlanckInstitut für extraterrestrische Physik (Germany); C. Goessl, F. Lang-Bardl, W. Mitsch, A. Riffeser, Univ.-Sternwarte München (Germany); N. Ageorges, Kayser-Threde GmbH (Germany)

$91452 \mathrm{E} \quad$ OAJ 2.6-m survey telescope: assembly, integration, and testing [9145-84]

O. Pirnay, C. Bastin, G. Lousberg, J.-M. Tortolani, P. Verheyden, AMOS Ltd. (Belgium)

TELESCOPE STRUCTURES, BEARINGS AND DRIVES

9145 2F Preliminary design study of the TMT Telescope structure system: overview [9145-86]

T. Usuda, National Astronomical Observatory of Japan (Japan); Y. Ezaki, N. Kawaguchi,

K. Nagae, A. Kato, J. Takaki, M. Hirano, T. Hattori, M. Tabata, Y. Horiuchi, Y. Saruta,

S. Sofuku, N. Itoh, T. Oshima, T. Takanezawa, M. Endo, Mitsubishi Electric Corp. (Japan);

J. Inatani, M. Iye, National Astronomical Observatory of Japan (Japan); A. Sadjadpour,

M. Sirota, S. Roberts, L. Stepp, Thirty Meter Telescope Observatory Corp. (United States)

$91452 \mathrm{G}$ New finite element models and seismic analyses of the telescopes at W.M. Keck Observatory [9145-87]

F. W. Kan, A. T. Sarawit, Simpson Gumpertz \& Heger Inc. (United States); S. P. Callahan, Caltech Optical Observatories (United States); M. L. Pollard, W. M. Keck Observatory (United States)

$91452 \mathrm{H} \quad$ Wheel drives for large telescopes: save the cost and keep the performance over hydrostatic bearings [9145-88]

M. F. Campbell, MF Campbell Consulting (United States)

$914521 \quad$ Finite element analyses of CCAT preliminary design [9145-89]

A. T. Sarawit, F. W. Kan, Simpson Gumpertz \& Heger Inc. (United States)

OBSERVATORY FACILITIES AND ENCLOSURES

9145 2J Approach to the E-ELT dome and main structure challenges [9145-90]

A. Bilbao, G. Murga, C. Gómez, J. Llarena, IDOM (Spain) 
9145 2K GMT site: facilities and enclosure design overview [9145-91]

J. Teran, M3 Engineering \& Technology Corp. (United States); M. Sheehan, Giant Magellan Telescope Organization Corp. (United States); D. H. Neff, E. Grigel, D. Adriaanse, M3 Engineering \& Technology Corp. (United States); A. Farahani, Giant Magellan Telescope Organization Corp. (United States)

$91452 \mathrm{~L}$ The Observatorio Astrofísico de Javalambre: engineering of observatory facilities and physical infrastructure, goals, and current status [9145-92]

A. Yanes-Díaz, R. Luis-Simoes, N. M. Lasso-Cabrera, S. Rueda-Teruel, F. Rueda-Tervel, J. L. Antón, S. Chueca, R. Bello, D. Jiménez-Mejías, O. Suárez, L. Guillén-Civera,

G. López-Alegre, M. A. C. Rodríguez-Hernández, M. Moles, A. J. Cenarro, A. Marin-Franch,

A. Ederoclite, D. Cristobal-Hornillos, J. Varela, T. Civera, D. Muniesa, H. Vazquez Ramió,

M. C. Díaz-Martín, R. Iglesias-Marzoa, N. Maicas, J. L. Lamadrid, A. Lopez-Sainz,

J. Hernández-Fuertes, L. Valdivielso, Ctr. de Estudios de Física del Cosmos de Aragón (Spain)

\section{MEASUREMENT AND CONTROL OF TELESCOPE VIBRATION}

$91452 \mathrm{M}$ VLTI-UT vibrations effort and performances [9145-93]

S. Poupar, P. Haguenauer, J. Alonso, N. Schuhler, J.-P. Henriquez, J.-P. Berger, P. Bourget,

S. Brillant, R. Castillo, P. Gitton, F. Gonte, European Southern Observatory (Chile); N. Di Lieto,

J.-L. Lizon, European Southern Observatory (Germany); A. Merand, European Southern

Observatory (Chile); J. Woillez, European Southern Observatory (Germany)

$91452 \mathrm{~N}$ Implementation of an active vibration damping system for the SOFIA telescope assembly [9145-94]

P. C. Janzen, P. J. Keas, Moog CSA Engineering (United States)

914520 Equipment vibration budget for the TMT [9145-95]

D. G. MacMartin, California Institute of Technology (United States) and Thirty Meter Telescope Observatory Corp. (United States); H. Thompson, Thirty Meter Telescope Observatory Corp. (United States)

POSTER SESSION

$91452 \mathrm{P} \quad$ Modeling and simulation of a 6-DOF parallel platform for telescope secondary mirror [9145-96]

Z. Yue, Y. Ye, National Astronomical Observatories, Nanjing Institute of Astronomical Optics \& Technology (China), Key Lab. of Astronomical Optics \& Technology, Nanjing Institute of Astronomical Optics \& Technology (China), and Univ. of Chinese Academy of Sciences (China); B. Gu, National Astronomical Observatories, Nanjing Institute of Astronomical Optics \& Technology (China) and Key Lab. of Astronomical Optics \& Technology, Nanjing Institute of Astronomical Optics \& Technology (China)

$91452 Q \quad$ Active optics operations at the Large Binocular Telescope [9145-97]

D. Miller, J. M. Hill, T. Golota, Large Binocular Telescope Observatory, The Univ. of Arizona (United States) 
9145 2R A laser tracker active optics system for the Large Binocular Telescope [9145-98] L. Dettmann, D. Ashby, J. M. Hill, A. Chatila, Large Binocular Telescope Observatory (United States)

$914525 \quad$ Active optics control development at the LBT [9145-99]

D. S. Ashby, C. J. Biddick, J. M. Hill, Large Binocular Telescope Observatory (United States)

$91452 \mathrm{~T}$ Challenges of the opto-mechanical conceptual design of a small far-IR balloon experiment [9145-100]

J.-L. Dournaux, Observatoire de Paris à Meudon, CNRS, Univ. Paris Diderot (France);

C. Berthod, Institut National des Sciences de l'Univ., CNRS (France); D. Horville, J.-M. Huet,

P. Laporte, Observatoire de Paris à Meudon, CNRS, Univ. Paris Diderot (France);

M. Wiedner, Observatoire de Paris, CNRS, Univ. Paris Diderot (France); A. Romanow,

Observatoire de Paris à Meudon, CNRS, Univ. Paris Diderot (France); J.-M. Krieg, L. Pagani, Observatoire de Paris, CNRS, Univ. Paris Diderot (France); J. Evrard, A. Gomes, M. Jouret,

CNES - Ctr. National d'Études Spatiales (France)

$91452 \mathrm{U} \quad$ Attitude determination for balloon-borne experiments [9145-101]

N. N. Gandilo, Univ. of Toronto (Canada); P. A. R. Ade, Cardiff Univ. (United Kingdom); M. Amiri, The Univ. of British Columbia (Canada); F. E. Angilè, Univ. of Pennsylvania (United States); S. J. Benton, Univ. of Toronto (Canada); J. J. Bock, California Institute of Technology (United States) and Jet Propulsion Lab. (United States); J. R. Bond, Canadian Institute for Theoretical Astrophysics (Canada) and Canadian Institute for Advanced Research (Canada); S. A. Bryan, Case Western Reserve Univ. (United States); H. C. Chiang, Univ. of Kwazulu-Natal (South Africa); C. R. Contaldi, Imperial College London (United Kingdom); B. P. Crill, California Institute of Technology (United States) and Jet Propulsion Lab. (United States); M. J. Devlin, B. Dober, Univ. of Pennsylvania (United States); O. P. Doré, California Institute of Technology (United States); M. Farhang, Univ. of Toronto (Canada) and Canadian Institute for Theoretical Astrophysics (Canada); J. P. Filippini, California Institute of Technology (United States); L. M. Fissel, Univ. of Toronto (Canada) and Northwestern Univ. (United States); A. A. Fraisse, Princeton Univ. (United States); Y. Fukui, Nagoya Univ. (Japan); N. Galitzki, Univ. of Pennsylvania (United States); A. E. Gambrel, Princeton Univ. (United States); S. Golwala, California Institute of Technology (United States);

J. E. Gudmundsson, Princeton Univ. (United States); M. Halpern, The Univ. of British Columbia (Canada) and Canadian Institute for Advanced Research (Canada); M. Hasselfield, Princeton Univ. (United States); G. C. Hilton, National Institute of Standards and Technology (United States); W. A. Holmes, Jet Propulsion Lab. (United States); V. V. Hristov, California Institute of Technology (United States); K. D. Irwin, National Institute of Standards and Technology (United States) and Stanford Univ. (United States); W. C. Jones, Z. D. Kermish, Princeton Univ. (United States); J. Klein, Univ. of Pennsylvania (United States); A. L. Korotkov, Brown Univ. (United States); C. L. Kuo, Stanford Univ. (United States); C. J. MacTavish, Kavli Institute for Cosmology, Univ. of Cambridge (United Kingdom); P. V. Mason, California Institute of Technology (United States); T. G. Matthews, Northwestern Univ. (United States); K. G. Megerian, Jet Propulsion Lab. (United States); L. Moncelsi, T. A. Morford, T. K. Mroczkowski, California Institute of Technology (United States); J. M. Nagy, Case Western Reserve Univ. (United States); C. B. Netterfield, Univ. of Toronto (Canada) and Canadian Institute for Advanced Research (Canada); G. Novak, Northwestern Univ. (United States); D. Nutter, Cardiff Univ. (United Kingdom); R. O'Brient, California Institute of Technology (United States) and Jet Propulsion Lab. (United States); E. Pascale, Cardiff Univ. (United Kingdom); F. Poidevin, Instituto de Astrofísica de Canarias (Spain) and Univ. de La Laguna (Spain); A. S. Rahlin, Princeton Univ. (United States); 
C. D. Reintsema, National Institute of Standards and Technology (United States); J. E. Ruhl, Case Western Reserve Univ. (United States); M. C. Runyan, Jet Propulsion Lab. (United States); G. Savini, Univ. College London (United Kingdom); D. Scott, The Univ. of British Columbia (Canada); J. A. Shariff, Univ. of Toronto (Canada); J. D. Soler, Univ. of Toronto (Canada) and Institut d'Astrophysique Spatiale, CNRS, Univ. Paris-Sud (France);

N. E. Thomas, Univ. of Miami (United States); A. Trangsrud, Jet Propulsion Lab. (United States); M. D. Truch, Univ. of Pennsylvania (United States); C. E. Tucker, Cardiff Univ. (United Kingdom); G. S. Tucker, Brown Univ. (United States); R. S. Tucker, California Institute of Technology (United States); A. D. Turner, Jet Propulsion Lab. (United States);

D. Ward-Thompson, Univ. of Central Lancashire (United Kingdom); A. C. Weber, Jet Propulsion Lab. (United States); D. V. Wiebe, The Univ. of British Columbia (Canada); E. Y. Young, Princeton Univ. (United States)

9145 2V Environmental testing for new SOFIA flight hardware [9145-103]

M. Lachenmann, J. Wolf, Deutsches SOFIA Institut, Univ. Stuttgart (Germany) and SOFIA Science Ctr., NASA Ames Research Ctr. (United States); R. Strecker, Deutsches SOFIA Institut, Univ. Stuttgart (Germany) and NASA Neil Armstrong Flight Research Ctr. (United States); B. Weckenmann, F. Trimpe, Deutsches SOFIA Institut, Univ. Stuttgart (Germany) and SOFIA Science Ctr., NASA Ames Research Ctr. (United States); H. J. Hall, SOFIA Science Ctr., Univ. Space Research Association (United States) and NASA Ames Research Ctr. (United States)

9145 2W BRRISON IR Camera (BIRC) [9145-105]

R. T. McMichael, M. W. Noble, D. Adams, P. Bernasconi, H. Borowski, Johns Hopkins Univ. Applied Physics Lab. (United States); R. Bupp, Nu-Tek Precision Optical Corp. (United States); A. F. Cheng, H. Eaton, R. Espiritu, Z. Fletcher, K. Heffernan, K. Hibbitts, C. L. Kee, G. Maahs, G. Murphy, D. Ponnusamy, N. W. Rolander, K. Strohbehn, J. R. Troll, B. D. Williams, Johns Hopkins Univ. Applied Physics Lab. (United States)

$91452 X \quad$ A prototype for the real-time analysis of the Cherenkov Telescope Array [9145-107] A. Bulgarelli, V. Fioretti, A. Zoli, INAF - IASF Bologna (Italy); A. Aboudan, INAF - IASF Bologna (Italy) and CISAS, Univ. degli Studi di Padova (Italy); J. J. Rodríguez-Vázquez, Ctr. de Investigaciones Energéticas, Medioambientales y Tecnológicas (Spain); G. Maier, Deutsched Elektronen-Synchrotron (Germany); E. Lyard, Univ. de Genève (Switzerland); D. Bastieri, Univ. degli Studi di Padova (Italy) and INAF - Osservatorio Astronomico di Roma (Italy); S. Lombardi, INAF - Osservatorio Astronomico di Roma (Italy); G. Tosti, Univ. degli Studi di Perugia (Italy); A. De Rosa, INAF - IASF Bologna (Italy); S. Bergamaschi, M. Interlandi, D. Beneventano, INAF - IASF Bologna (Italy) and Univ. degli Studi di Modena e Reggio Emilia (Italy); G. Lamanna, J. Jacquemier, Lab. d'Annecy-le-Vieux de Physique des Particules, CNRS, Univ. de Savoie (France); K. Kosack, IRFU-CEA (France); L. A. Antonelli, INAF - Osservatorio Astronomico di Roma (Italy); C. Boisson, Observatoire de Paris, CNRS, Univ. Paris Diderot (France); J. Burkowski, Copernicus Astronomical Ctr. (Poland); S. Buson, Univ. degli studi di Padua (Italy); A. Carosi, INAF - Osservatorio Astronomico di Roma (Italy); V. Conforti, INAF - IASF Bologna (Italy); J. L. Contreras, Univ. Complutense de Madrid (Spain); G. De Cesare, INAF - IASF Bologna (Italy); R. de los Reyes, Max-Planck-Institut für Kernphysik (Germany); J. Dumm, Univ. of lowa (United States); P. Evans, Univ. of Leicester (United Kingdom); L. Fortson, Univ. of lowa (United States); M. Fuessling, Univ. Potsdam (Germany); R. Graciani, Univ. de Barcelona (Italy); F. Gianotti, P. Grandi INAF - IASF Bologna (Italy); J. Hinton, Univ. of Leicester (United Kingdom); B. Humensky, Columbia Univ. (United States); J. Knödlseder, Institut de Recherche en Astrophysique et Planétologie, CNRS (France); G. Malaguti, M. Marisaldi, INAF - IASF Bologna (Italy); N. Neyroud, Lab. d'Annecy-le-Vieux de Physique des Particules, CNRS, Univ. de Savoie (France); L. Nicastro, 
INAF - IASF Bologna (Italy); S. Ohm, J. Osborne, S. Rosen, Univ. of Leicester (United Kingdom); A. Tacchini, E. Tossesi, INAF - IASF Bologna (Italy); V. Testa, INAF - Osservatorio Astronomico di Roma (Italy); M. Trifoglio, INAF - IASF Bologna (Italy); A. Weinstein, lowa State Univ. (United States)

$91452 Y$ SST-GATE telescope: an innovative dual-mirror prototype for the Cherenkov Telescope Array [9145-108]

D. Dumas, J.-M. Huet, J.-L. Dournaux, P. Laporte, J.-P. Amans, G. Fasola, A. Zech, C. Rulten, H. Sol, Observatoire de Paris à Meudon, CNRS, Univ. Paris Diderot (France); S. Blake, J. Schmoll, Durham Univ. (United Kingdom)

$91452 Z$ SST dual-mirror telescopes for the Cherenkov Telescope Array [9145-109] D. Dumas, P. Laporte, H. Sol, Observatoire de Paris à Meudon, CNRS, Univ. Paris Diderot (France); G. Pareschi, R. Canestrari, INAF - Osservatorio Astronomico di Brera (Italy); L. Stringhetti, INAF - IASF Milano (Italy); O. Catalano, INAF - IASF Palermo (Italy); R. White, T. Greenshaw, J. Hinton, Univ. of Leicester (United Kingdom); S. Blake, Durham Univ. (United Kingdom)

914530 Performance of the small size telescope sub-array of the Cherenkov Telescope Array observatory [9145-110]

R. Moderski, Nicolaus Copernicus Astronomical Ctr. (Poland); J. A. Aguilar, Univ. de Genève (Switzerland); W. Bilnik, AGH Univ. of Science and Technology (Poland); L. Bogacz, Jagiellonian Univ. (Poland); T. Bulik, Univ. of Warsaw (Poland); A. Christov, D. della Volpe, Univ. de Genève (Switzerland); M. Dyrda, Institute of Nuclear Physics (Poland);

A. Frankowski, Nicolaus Copernicus Astronomical Ctr. (Poland); M. Grudzińska, Univ. of Warsaw (Poland); J. Grygorczuk, Space Research Ctr. (Poland); M. Heller, Univ. de Genève (Switzerland); B. Idźkowski, Astronomical Observatory, Jagiellonian Univ. (Poland); M. Janiak, Nicolaus Copernicus Astronomical Ctr. (Poland); M. Jamrozy, Astronimical Observatory, Jagiellonian Univ. (Poland); M. Karczewski, Space Research Ctr. (Poland); J. Kasperek, AGH Univ. of Science and Technology (Poland); E. Lyard, Univ. de Genève (Switzerland); A. Marszałek, Astronomical Observatory, Jagiellonian Univ. (Poland); J. Michałowski, Institute of Nuclear Physics (Poland); R. Mohamed, T. Montaruli, A. Neronov, Univ. de Genève (Switzerland); J. Nicolau-Kukliński, Space Research Ctr. (Poland); J. Niemiec, Institute of Nuclear Physics (Poland); M. Ostrowski, Astronomical Observatory, Jagiellonian Univ. (Poland); P. Paśko, Ł. Płałos, Space Research Ctr. (Poland); E. Prandini, Univ. de Genève (Switzerland); R. Pruchniewicz, AGH Univ. of Science and Technology (Poland); J. Rafalski, Institute of Nuclear Physics (Portugal); P. J. Rajda, AGH Univ. of Science and Technology (Poland); M. Rataj, Space Research Ctr. (Poland); M. Rupiński, AGH Univ. of Science and Technology (Poland); K. Rutkowski, K. Seweryn, M. Sidz, Space Research Ctr. (Poland); L. Stawarz, M. Stodulska, Astronomical Observatory, Jagiellonian Univ. (Poland); M. Stodulski, Institute of Nuclear Physics (Poland); M. Tokarz, Space Research Ctr. (Poland); S. Toscano, I. Troyano Pujadas, R. Walter, Univ. de Genève (Switzerland); P. Wawer, R. Wawrzaszek, L. Wiśniewski, Space Research Ctr. (Poland); K. Ziętara, Astronomical Observatory, Jagiellonian Univ. (Poland); P. Ziółkowski, P. Żychowski, Institute of Nuclear Physics (Poland)

914531 Status of the photomultiplier-based FlashCam camera for the Cherenkov Telescope Array [9145-112]

G. Pühlhofer, Eberhard-Karls-Univ. (Germany); C. Bauer, Max-Planck-Institut für Kernphysik (Germany); F. Eisenkolb, Eberhard-Karls-Univ. (Germany); D. Florin, Univ. Zürich

(Switzerland); C. Föhr, Max-Planck-Institut für Kernphysik (Germany); A. Gadola, Univ. Zürich

(Switzerland); F. Garrecht, G. Hermann, Max-Planck-Institut für Kernphysik (Germany); 
I. Jung, O. Kalekin, Friedrich-Alexander-Univ. Erlangen-Nürnberg (Germany); C. Kalkuhl, Eberhard-Karls-Univ. (Germany); J. Kasperek, AGH Univ. of Science and Technology (Poland); T. Kihm, Max-Planck-Institut für Kernphysik (Germany); J. Koziol, Jagiellonian Univ. (Poland); R. Lahmann, Friedrich-Alexander-Univ. Erlangen-Nürnberg (Germany); A. Manalaysay, Univ. Zürich (Switzerland); A. Marszalek, Jagiellonian Univ. (Poland); P. J. Rajda, AGH Univ. of Science and Technology (Poland); O. Reimer, Univ. of Innsbruck (Austria); W. Romaszkan, M. Rupinski, AGH Univ. of Science and Technology (Poland); T. Schanz, Eberhard-Karls-Univ. (Germany); T. Schwab, Max-Planck-Institut für Kernphysik (Germany); S. Steiner, U. Straumann, Univ. Zürich (Switzerland); C. Tenzer, Eberhard-KarlsUniv. (Germany); A. Vollhardt, Univ. Zürich (Switzerland); Q. Weitzel, Max-Planck-Institut für Kernphysik (Germany); K. Winiarski, AGH Univ. of Science and Technology (Poland); K. Zietara, Jagiellonian Univ. (Poland)

914533 Schwarzschild-Couder Telescope for The Cherenkov Telescope Array [9145-114] K. J. Meagher, Georgia Institute of Technology (United States)

914534 Thermal design and performance of the balloon-borne large aperture submillimeter telescope for polarimetry BLASTPol [9145-116]

J. D. Soler, Institut d'Astrophysique Spatiale, CNRS (France) and Univ. of Toronto (Canada); P. A. R. Ade, Cardiff Univ. (United Kingdom); F. E. Angilè, Univ. of Pennsylvania (United States); S. J. Benton, Univ. of Toronto (Canada); M. J. Devlin, B. Dober, Univ. of Pennsylvania (United States); L. M. Fissel, Institut d'Astrophysique Spatiale, CNRS (France) and Northwestern Univ. (United States); Y. Fukui, Nagoya Univ. (Japan); N. Galitzki, Univ. of Pennsylvania (United States); N. N. Gandilo, Univ. of Toronto (Canada); J. Klein, Univ. of Pennsylvania (United States); A. L. Korotkov, Brown Univ. (United States); T. G. Matthews, Northwestern Univ. (United States); L. Moncelsi, California Institute of Technology (United States); A. Mroczkowski, US Naval Research Lab. (United States); C. B. Netterfield, Univ. of Toronto (Canada) and Canadian Institute for Advanced Research (Canada); G. Novak, Northwestern Univ. (United States); D. Nutter, E. Pascale, Cardiff Univ. (United Kingdom); F. Poidevin, Instituto de Astrofísica de Canarias (Spain); G. Savini, Univ. College London (United Kingdom); D. Scott, The Univ. of British Columbia (Canada); J. A. Shariff, Univ. of Toronto (Canada); N. E. Thomas, Univ. of Miami (United States); M. D. Truch, Univ. of Pennsylvania (United States); C. E. Tucker, Cardiff Univ. (United Kingdom); G. S. Tucker, Brown Univ. (United States); D. Ward-Thompson, Jeremiah Horrocks Institute of Maths, Physics, and Astronomy (United Kingdom)

914535 Development of a 30-cm submillimeter-wave telescope for the operation at Dome Fuji in Antarctica [9145-1 17]

S. Ishii, Univ. of Tsukuba (Japan) and The Univ. of Tokyo (Japan); M. Seta, T. Nagasaki, N. Nakai, M. Nagai, Univ. of Tsukuba (Japan); Y. Miyamoto, Ibaraki Univ. (Japan); H. Imada, K. Doihata, K. Saito, Univ. of Tsukuba (Japan); Y. Sekimoto, National Astronomical Observatory of Japan (Japan)

914538 Wide field corrector for the KMTNet telescope [9145-119]

Y. Lee, S.-M. Cha, Korea Astronomy and Space Science Institute (Korea, Republic of) and Kyung Hee Univ. (Korea, Republic of); W. Poteet, CP Systems, Inc. (United States); P. Lam, Lam Optics, Inc. (United States); C.-U. Lee, S.-L. Kim, B.-G. Park, Korea Astronomy and Space Science Institute (Korea, Republic of); R. A. Buchroeder, CP Systems, Inc. (United States); H. Jin, Kyung Hee Univ. (Korea, Republic of) 
914539 Stress polishing demonstrator for ELT M1 segments and industrialization [9145-120] E. Hugot, A. Bernard, Lab. d'Astrophysique de Marseille (France); M. Laslandes, Space Structures Lab., California Institute of Technology (United States); J. Floriot, Lab. d'Astrophysique de Marseille (France); T. Dufour, D. Fappani, J. M. Combes, Thales SESO (France); M. Ferrari, Lab. d'Astrophysique de Marseille (France)

9145 3A The INdoor turbulENce SEnsor (INTENSE) instrument [9145-121] J. Chabé, F. Blary, A. Ziad, J. Borgnino, Y. Fantei-Caujolle, Observatoire de la Côte d'Azur, CNRS, Univ. de Nice-Sophia Antipolis (France); A. Liotard, F. Falzon, Thales Alenia Space (France)

\section{Part Three}

9145 3B Approaches to the interferometric test of large flat mirrors: the case of the adaptive M4 for E-ELT [9145-123]

G. Pariani, INAF - Osservatorio Astronomico di Brera (Italy); R. Briguglio, M. Xompero,

A. Riccardi, INAF - Osservatorio Astrofisico di Arcetri (Italy); M. Riva, A. Bianco, F. M. Zerbi, INAF - Osservatorio Astronomico di Brera (Italy); D. Tresoldi, INAF - IASF Milano (Italy); E. Molinari, INAF - Fundación Galileo Galilei (Spain); M. Tintori, P. Lazzarini, D. Gallieni, A.D.S. International S.r.l. (Italy); R. Biasi, Microgate (Italy); E. Vernet, M. Cayrel, European Southern Observatory (Germany)

$91453 \mathrm{C}$ Design and development status of the University of Tokyo Atacama Observatory 6.5m telescope [9145-124]

T. Morokuma, The Univ. of Tokyo (Japan); T. Aoki, Kiso Observatory, The Univ. of Tokyo (Japan); M. Doi, The Univ. of Tokyo (Japan); T. Handa, Kagoshima Univ. (Japan); T. Kamizuka, N. Kato, K. Kawara, K. Kohno, M. Konishi, The Univ. of Tokyo (Japan); S. Koshida, Pontificia Univ. Católica de Chile (Chile); T. Minezaki, T. Miyata, K. Motohara, S. Sako, The Univ. of Tokyo (Japan); T. Soyano, Kiso Observatory, The Univ. of Tokyo (Japan); H. Takahashi, Y. Tamura, T. Tanabé, M. Tanaka, The Univ. of Tokyo (Japan); K. Tarusawa, Kiso Observatory, The Univ. of Tokyo (Japan); Y. Yoshii, The Univ. of Tokyo (Japan)

9145 3D Progress on the New York State Observatory: a new 12-meter astronomical telescope [9145-125]

T. Sebring, Xoptx LLC (United States); C. O'Dea, S. Baum, Rochester Institute of Technology (United States); J. Teran, M3 Engineering \& Technology Corp. (United States); N. Loewen, Dynamic Structures Ltd. (Canada); C. Stutzki, Stutzki Engineering, Inc. (United States); R. Egerman, Exelis Corp. (United States); G. Bonomi, Ingersoll Machines Tools, Inc. (United States)

9145 3E Astroclimate at Jbel Aklim site in Moroccan anti-atlas: 2008-2010 seeing and isoplanatic angle statistics from the E-ELT site testing data [9145-126]

M. Sabil, Z. Benkhaldoun, M. Lazrek, A. Habib, Y. Hach, A. Benhida, A. Jabiri, Y. Elazhari, Univ. Cadi Ayad Marrakech (Morocco) 
9145 3F E-ELT seeing and isoplanatic angle: comparison of Aklim site and El Roque de Los Muchachos Observatory [9145-127]

M. Sabil, Z. Benkhaldoun, M. Lazrek, A. Habib, Univ. Cadi Ayad Marrakech (Morocco); A. Benhida, Univ. Cadi Ayad Marrakech (Morocco) and Ctr. National des Innovations Pédagogiques et d'Expérimentation (Morocco); Y. Hach, Univ. Cadi Ayad Marrakech (Morocco); Y. Elazhari, Univ. Cadi Ayad Marrakech (Morocco) and Ctr. National des Innovations Pédagogiques et d'Expérimentation (Morocco); T. Elhalkouj, Univ. Cadi Ayad Marrakech (Morocco)

$91453 G \quad$ A new generalized differential image motion monitor [9145-128]

E. Aristidi, Y. Fanteï-Caujolle, A. Ziad, C. Dimur, J. Chabé, B. Roland, Lab. Lagrange, Observatoire de la Côte d'Azur, CNRS, Univ. of Nice Sophia-Antipolis (France)

$91453 \mathrm{H} \quad$ Monitoring atmospheric turbulence profiles with high vertical resolution using PML/PBL instrument [9145-129]

F. Blary, A. Ziad, J. Borgnino, Y. Fantéï-Caujolle, E. Aristidi, H. Lantéri, Lab. Lagrange, Observatoire de la Côte d'Azur, CNRS, Univ. of Nice Sophia-Antipolis (France)

$914531 \quad$ Phase characteristics of the ALMA 3-km baseline data [9145-130]

S. Matsushita, Institute of Astronomy and Astrophysics, Academia Sinica (Taiwan); Y. Asaki, Institute of Space and Astronautical Science (Japan) and The Graduate Univ. for Advanced Studies (Japan); R. Kawabe, National Astronomical Observatory of Japan (Japan); E. Fomalont, National Radio Astronomy Observatory (United States) and Joint ALMA Observatory (Chile); D. Barkats, S. Corder, Joint ALMA Observatory (Chile)

9145 3J Optical turbulence profiling with SIODAR in the Canadian High Arctic [9145-131] J. Maire, E. Mieda, Univ. of Toronto (Canada); E. Steinbring, R. Murowinski, National Research Council Canada (Canada); J. R. Graham, Univ. of Toronto (Canada) and Univ. of California, Berkeley (United States); R. Carlberg, S. A. Wright, Univ. of Toronto (Canada); N. M. Law, Univ. of Toronto (Canada) and The Univ. of North Carolina at Chapel Hill (United States); S. Sivanandam, Univ. of Toronto (Canada)

9145 3K SLODAR instrument for characterizing an arctic site: overview of the experimental method, design, and performance [9145-132]

E. Mieda, Univ. of Toronto (Canada) and Dunlap Institute for Astronomy and Astrophysics (Canada); J. Maire, Dunlap Institute for Astronomy and Astrophysics (Canada);

J. R. Graham, Univ. of California, Berkeley (United States); S. A. Wright, Univ. of Toronto (Canada) and Dunlap Institute for Astronomy and Astrophysics (Canada); D.-S. Moon, Univ. of Toronto (Canada)

9145 3L Calibration systems for LSST [9145-133]

J. Sebag, W. Gressler, M. Liang, T. Axelrod, C. Claver, J. Andrew, National Optical Astronomy Observatory (United States)

9145 3M Reliability-centered maintenance for ground-based large optical telescopes and radio antenna arrays [9145-135]

G. Marchiori, F. Formentin, F. Rampini, European Industrial Engineering s.r.l. (Italy) 
$91453 \mathrm{~N}$ Wavefront coding applied to a two-mirror telescope [9145-136]

J. A. Araiza-Duran, Instituto Nacional de Astrofísica, Óptica y Electrónica (Mexico); E. Luna, Instituto de Astronomía, Univ. Nacional Autónoma de México (Mexico); A. Cornejo, Instituto Nacional de Astrofísica, Óptica y Electrónica (Mexico); J. Herrera, Univ. Nacional Autónoma de México (Mexico)

$91453 \mathrm{P} \quad$ Three wide-field telescopes with spherical primary mirrors [9145-138] D. Blanco, MMT Observatory, Univ. of Arizona (United States)

$91453 Q$ Simultaneous ultra-high contrast imaging and determination of time-dependent, noncommon path aberrations in the presence of detector noise [9145-139]

R. A. Frazin, Univ. of Michigan (United States)

$91453 R \quad$ Integration and commissioning of the China SONG Telescope Tracking System [9145-140] C. Ren, H. Wan, National Astronomical Observatories, Nanjing Institute of Astronomical Optics \& Technology (China); H. Li, National Astronomical Observatories, Nanjing Institute of Astronomical Optics \& Technology (China) and Graduate Univ. of Chinese Academy of Sciences (China); J. Xu, National Astronomical Observatories, Nanjing Institute of Astronomical Optics \& Technology (China); G. Wang, National Astronomical Observatories, Nanjing Institute of Astronomical Optics \& Technology (China) and The Key Lab. of Astronomical Optics \& Technology Technology, Nanjing Institute of Astronomical Optics \& Technology (China); X. Jiang, National Astronomical Observatories, Nanjing Institute of Astronomical Optics \& Technology (China)

914535 Fly's Eye camera system: optical imaging using a hexapod platform [9145-141] A. Jaskó, MTA Research Ctr. for Astronomy and Earth Sciences (Hungary); A. Pál, MTA Research Ctr. for Astronomy and Earth Sciences (Hungary) and Eötvös Loránd Univ. (Hungary); K. Vida, MTA Research Ctr. for Astronomy and Earth Sciences (Hungary); L. Mészáros, MTA Research Ctr. for Astronomy and Earth Sciences (Hungary) and Eötvös Loránd Univ. (Hungary); G. L. Csépány, MTA Research Ctr. for Astronomy and Earth Sciences (Hungary), Eötvös Loránd Univ. (Hungary), and European Southern Observatory (Germany); G. Mező, MTA Research Ctr. for Astronomy and Earth Sciences (Hungary)

$91453 \mathrm{~T}$ Observational performance of the KMTNet [9145-142]

C.-U. Lee, S.-L. Kim, S.-M. Cha, Y. Lee, D.-J. Kim, B.-G. Park, Korea Astronomy and Space Science Institute (Korea, Republic of)

9145 3V The PANOPTES project: discovering exoplanets with low-cost digital cameras [9145-145] O. Guyon, Subaru Telescope, National Astronomical Observatory of Japan (United States) and Steward Observtory, The Univ. of Arizona (United States); J. Walawender, N. Jovanovic, Subaru Telescope, National Astronomical Observatory of Japan (United States); M. Butterfield, The College of Optical Sciences, The Univ. of Arizona (United States); W. T. Gee, Subaru Telescope, National Astronomical Observatory of Japan (United States) and The Univ. of Hawaili at Hilo (United States); R. Mery, Institut d'Optique (France)

9145 3W Dome shutter failure causes longest shutdown (67-nights) ever recorded by the CFHT Observatory [9145-146]

I. A. Look, D. Salmon, S. Bauman, K. Ho, C. Elizares, Canada-France-Hawaii Telescope (United States) 
$91453 X \quad$ Design development of a deployable tertiary mirror for Keck [9145-147]

J. X. Prochaska, J. Cabak, C. Ratliff, S. Adkins, M. Bolte, D. Cowley, H. Epps, J. Nelson,

A. Tripsas, A. C. Phillips, W. Deich, M. Peck, Univ. of California Observatories (United States)

$91453 Y \quad$ Planning the installation of the dark energy spectroscopic instrument on the Mayall Telescope [9145-148]

D. Sprayberry, National Optical Astronomy Observatory (United States); W. Goble, MMT Observatory, Univ. of Arizona (United States); L. Allen, J. Elias, R. Probst, R. Joyce, A. Dey, R. Marshall, M. Evatt, R. Blum, National Optical Astronomy Observatory (United States); T. M. C. Abbott, A. Walker, F. Muñoz, National Optical Astronomy Observatory (Chile); R. Besuner, Space Science Lab., Univ. of California, Berkeley (United States); M. Sholl, Google, Inc. (United States); P. Jelinsky, J. Silber, Space Science Lab., Univ. of California, Berkeley (United States); R. Lafever, C. Bebek, Lawrence Berkeley National Lab. (United States); B. Flaugher, Fermi National Accelerator Lab. (United States)

$91453 Z$ A laser guide star system for LAMOST [9145-149]

H. Bai, X. Yuan, M. Liang, Nanjing Institute of Astronomical Optics and Technology (China)

914540 Upgradation progress of $13.7 \mathrm{~m}$ millimeter radio telescope reflector surface maintenance test [9145-150]

Y. Zhang, D. Yang, G. Zhou, Y. Wang, National Astronomical Observatories, Nanjing Institute of Astronomical Optics \& Technology (China) and Key Lab. of Astronomical Optics \& Technology, Nanjing Institute of Astronomical Optics \& Technology (China); A. Li, National Astronomical Observatories, Nanjing Institute of Astronomical Optics \& Technology (China), Key Lab. of Astronomical Optics \& Technology, Nanjing Institute of Astronomical Optics \& Technology (China), and Univ. of Chinese Academy of Sciences (China); Y. Li, A. Liu, Y. Zeng, G. Li, National Astronomical Observatories, Nanjing Institute of Astronomical Optics \& Technology (China) and Key Lab. of Astronomical Optics and Technology (China); Q. Wang, National Astronomical Observatories, Nanjing Institute of Astronomical Optics \& Technology (China), Key Lab. of Astronomical Optics \& Technology, Nanjing Institute of Astronomical Optics \& Technology (China), and Univ. of Chinese Academy of Sciences (China)

914542 Save our secondary: recovering a broken 1.3-m mirror [9145-152]

T. M. C. Abbott, Cerro Tololo Inter-American Observatory (Chile); R. G. Probst, G. Poczulp, National Optical Astronomy Observatory (United States); R. Tighe, P. Schurter, A. Montané, Cerro Tololo Inter-American Observatory (Chile); J. DeVries, R. C. Harris, J. Elias, National Optical Astronomy Observatory (United States); M. Martinez, O. Saa, Cerro Tololo InterAmerican Observatory (Chile)

914543 The Mercator telescope: relevance, status, and future [9145-153]

G. Raskin, W. Pessemier, Institute of Astronomy, Katholieke Univ. Leuven (Belgium); F. Merges, J. Pérez Padilla, S. Prins, Mercator Telescope, Roque de Los Muchachos Observatory (Spain); H. Van Winckel, Institute of Astronomy, Katholieke Univ. Leuven (Belgium)

914544 Concept and implementation of a virtual image slicer at the VLT [9145-154]

S. Guisard, European Southern Observatory (Chile); M. Sterzik, European Southern Observatory (Germany); I. Muñoz, European Southern Observatory (Chile) 
914546 Laboratory performance testing, installation, and commissioning of the wide field upgrade tracker for the Hobby-Eberly Telescope [9145-156]

J. Good, J. Booth, M. E. Cornell, G. J. Hill, H. Lee, R. Savage, R. Leck, H. Kriel, M. Landriau, McDonald Observatory, The Univ. of Texas at Austin (United States)

914547 Turkey's next big science project: DAG the 4 meter telescope [9145-210]

O. Keskin, Isik Univ. (Turkey); C. Yesilyaprak, Ataturk Univ. (Turkey); S. K. Yerli, Orta Dogu Teknik Univ. (Turkey); L. Zago, L. Jolissaint, Haute Ecole d'Ingénierie et de Gestion du Canton de Vaud (Switzerland)

914549 Challenges in operating an Arctic telescope [9145-212]

L. Ivănescu, Univ. de Sherbrooke (Canada) and Univ. du Québec à Montréal (Canada);

K. Baibakov, N. T. O'Neill, Univ. de Sherbrooke (Canada); J.-P. Blanchet, Univ. du Québec à Montréal (Canada); Y. Blanchard, A. Saha, Univ. de Sherbrooke (Canada); M. Rietze, Baader Planetarium GmbH (Germany); K.-H. Schulz, Dr. Schulz \& Partner GmbH (Germany)

9145 4A LSST telescope integration and tests [9145-157]

J. Sebag, W. Gressler, D. Neill, J. Barr, C. Claver, J. Andrew, National Optical Astronomy Observatory (United States)

9145 4B Tuning a 2.4-meter telescope... blindfolded [9145-158]

K. Lanclos, M. Peck, M. Saylor, R. I. Kibrick, S. L. Allen, Univ. of California Observatories, Santa Cruz (United States)

9145 4C Design and implementation of coating hardware for the Hobby-Eberly Telescope widefield corrector [9145-160]

J. Good, H. Lee, G. J. Hill, B. Vattiat, D. Perry, H. Kriel, R. Savage, McDonald Observatory, The Univ. of Texas at Austin (United States)

9145 4E SPEED: the segmented pupil experiment for exoplanet detection [9145-162] P. Martinez, O. Preis, C. Gouvret, J. Dejonghe, J.-B. Daban, A. Spang, F. Martinache, M. Beaulieu, P. Janin-Potiron, L. Abe, Y. Fantei-Caujolle, D. Mattei, S. Ottogalli, Lab. Lagrange, Observatoire de la Côte d'Azur, CNRS, Univ. of Nice Sophia-Antipolis (France)

$91454 \mathrm{~F}$ The integrated motion measurement simulation for SOFIA [9145-164] P. Kaswekar, B. Greiner, J. Wagner, Univ. Stuttgart (Germany)

9145 4G Measuring transmission and forces from observatory equipment vibration [9145-166] H. Thompson, Thirty Meter Telescope Observatory Corp. (United States); D. G. MacMartin, Thirty Meter Telescope Observatory Corp. (United States) and California Institute of Technology (United States); P. W. G. Byrnes, National Research Council Canada (Canada); D. Tomono, H. Terada, Subaru Telescope, National Astronomical Observatory of Japan (United States)

$91454 \mathrm{H} \quad$ Accelerometer-based position reconstruction for the feedforward compensation of fast telescope vibrations in the E-ELT/MICADO [9145-167]

A. Keck, Univ. Stuttgart (Germany) and Max-Planck-Institut für Astronomie (Germany); J.-U. Pott, Max-Planck-Institut für Astronomie (Germany); O. Sawodny, Univ. Stuttgart (Germany) 
91454 ALMA band 10 (787-950 GHz) first astronomical fringes [9145-168]

S. Asayama, Joint ALMA Observatory (Chile) and National Astronomical Observatory of Japan (Japan); L. B. G. Knee, National Research Council Canada (Canada); P. G. Calisse, Joint ALMA Observatory (Chile) and European Southern Observatory (Chile); J. P. Colque, C. M. Lopez, Joint ALMA Observatory (Chile); T. Nakos, N. M. Phillips, Joint ALMA Observatory (Chile) and European Southern Observatory (Chile); K. H. Plarre, M. C. Radiszcz, Joint ALMA Observatory (Chile); G. Siringo, N. D. Whyborn, Joint ALMA Observatory (Chile) and European Southern Observatory (Chile); H. Yatagai, Joint ALMA Observatory (Chile) and National Astronomical Observatory of Japan (Japan)

$91454 \mathrm{~J}$ A holography receiver for the Atacama Large millimeter/submillimeter array antenna and the measured surface deformation [9145-169]

H. Kiuchi, M. Yamada, M. Sugimoto, M. Saito, National Astronomical Observatory of Japan (Japan)

9145 4K ALMA fast switching phase calibration on long baselines [9145-170] Y. Asaki, Institute of Space and Astronautical Science, Japan Aerospace Exploration Agency (Japan) and Japan Aerospace Exploration Agency (Japan); S. Matsushita, Academia Sinica Institute of Astronomy and Astrophysics (Taiwan); R. Kawabe, National Astronomical Observatory of Japan (Japan); E. Fomalont, National Radio Astronomy Observatory (United States) and Joint ALMA Observatory (Chile); D. Barkats, S. Corder, Joint ALMA Observatory (Chile)

9145 4L GMT enclosure structure and mechanism design [9145-171] J. Teran, M3 Engineering \& Technology Corp. (United States); M. Sheehan, Giant Magellan Telescope Organization Corp. (United States); D. H. Neff, N. Korde, E. Manuel, A. Ortega, M3 Engineering \& Technology Corp. (United States)

9145 4M Building information modeling (BIM) approach to the GMT Project [9145-172] J. Teran, M3 Engineering \& Technology Corp. (United States); M. Sheehan, Giant Magellan Telescope Organization Corp. (United States); D. H. Neff, D. Adriaanse, E. Grigel, M3 Engineering \& Technology Corp. (United States); A. Farahani, Giant Magellan Telescope Organization Corp. (United States)

$91454 \mathrm{~N}$ Design of mirror coating facility for The University of Tokyo Atacama Observatory $6.5 \mathrm{~m}$ telescope [9145-173]

H. Takahashi, The Univ. of Tokyo (Japan); T. Aoki, The Univ. of Tokyo (Japan) and Kiso Observatory, The Univ. of Tokyo (Japan); M. Doi, The Univ. of Tokyo (Japan); T. Handa, Kagoshima Univ. (Japan); T. Kamizuka, N. Kato, K. Kawara, K. Kohno, M. Konishi, The Univ. of Tokyo (Japan); S. Koshida, Pontificia Univ. Católica de Chile (Chile); T. Minezaki, T. Miyata, T. Morokuma, K. Motohara, S. Sako, The Univ. of Tokyo (Japan); T. Soyano, The Univ. of Tokyo (Japan) and Kiso Observatory, The Univ. of Tokyo (Japan); Y. Tamura, T. Tanabé, M. Tanaka, The Univ. of Tokyo (Japan); K. Tarusawa, The Univ. of Tokyo (Japan) and Kiso Observatory, The Univ. of Tokyo (Japan); Y. Yoshii, The Univ. of Tokyo (Japan)

914540 Baseline design and requirements for the LSST rotating enclosure (dome) [9145-174] D. R. Neill, J. DeVries, E. Hileman, J. Sebag, W. Gressler, O. Wiecha, J. Andrew, W. Schoening, National Optical Astronomy Observatory (United States) 
$91454 \mathrm{P} \quad$ Design of enclosure and support facilities for The University of Tokyo Atacama Observatory 6.5-m Telescope [9145-175]

S. Sako, Y. Yoshii, M. Doi, K. Kohno, T. Miyata, K. Motohara, K. Kawara, M. Tanaka, T. Minezaki, T. Morokuma, Y. Tamura, T. Tanabe, H. Takahashi, M. Konishi, T. Kamizuka, The Univ. of Tokyo (Japan); S. Koshida, The Univ. of Tokyo (Japan) and Pontificia Univ. Católica de Chile (Chile); N. Kato, The Univ. of Tokyo (Japan); T. Aoki, T. Soyano, K. Tarusawa, Kiso Observatory, The Univ. of Tokyo (Japan); T. Handa, Kagoshima Univ. (Japan); L. Bronfman, M. T. Ruiz, M. Hamuy, R. Mendez, Univ. of Chile (Chile)

9145 4Q SKA mid-frequency aperture array thermal analysis for South Africa [9145-176]

H. Hanenburg, ASTRON (Netherlands); J. Kragt, NOVA, ASTRON (Netherlands); R. v. d. Brink, M. Drost, ASTRON (Netherlands)

$91454 R \quad$ The SRT inclinometer for monitoring the rail and the thermal gradient effects on the alidade structure [9145-178]

T. Pisanu, F. Buffa, S. Poppi, P. Marongiu, G. Serra, G. P. Vargiu, R. Concu, INAF -

Osservatorio Astronomico di Cagliari (Italy)

$91454 S$ Sardinia aperture array demonstrator [9145-179]

M. Murgia, INAF - Osservatorio Astronomico di Cagliari (Italy); G. Bianchi, INAF - Istituto di Radiastronomia di Bologna (Italy); P. Bolli, G. Comoretto, INAF - Osservatorio Astrofisico di Arcetri (Italy); D. Dallacasa, Univ. degli studi di Bologna (Italy); M. Z. Farooqui, Istituto di Elettronica e Ingegneria dell'Informazione Torino, CNR (Italy); F. Gaudiomonte, INAF Istituto di Radiastronomia di Bologna (Italy); L. Gregorini, Univ. di Bologna (Italy); F. Govoni, INAF - Osservatorio Astronomico di Cagliari (Italy); K.-H. Mack, M. Massardi, A. Mattana, INAF - Istituto di Radiastronomia di Bologna (Italy); A. Melis, INAF - Osservatorio Astronomico di Cagliari (Italy); J. Monari, INAF - Istituto di Radiastronomia di Bologna (Italy); L. Mureddu, INAF - Osservatorio Astronomico di Cagliari (Italy); G. Naldi, INAF - Istituto di Radiastronomia di Bologna (Italy); F. Paonessa, Istituto di Elettronica e Ingegneria dell'Informazione Torino, CNR (Italy); F. Perini, INAF - Istituto di Radiastronomia di Bologna (Italy); A. Poddighe, I. Porceddu, INAF - Osservatorio Astronomico di Cagliari (Italy); I. Prandoni, G. Pupillo, M. Schiaffino, F. Schillirò, INAF - Istituto di Radiastronomia di Bologna (Italy); G. Serra, INAF Osservatorio Astronomico di Cagliari (Italy); A. Tibaldi, Istituto di Elettronica e Ingegneria dell'Informazione Torino, CNR (Italy); T. Venturi, INAF - Istituto di Radiastronomia di Bologna (Italy); G. Virone, Istituto di Elettronica e Ingegneria dell'Informazione Torino, CNR (Italy); A. Zanichelli, INAF - Istituto di Radiastronomia di Bologna (Italy)

9145 4T QUIJOTE-CMB experiment: a technical overview [9145-180]

A. R. Pérez-de-Taoro, M. Aguiar-González, R. Génova-Santos, F. Gómez-Reñasco, R. Hoyland, C. López-Caraballo, A. Peláez-Santos, F. Poidevin, D. Tramonte, R. Rebolo-López, J. A. Rubiño-Martín, V. Sánchez-de la Rosa, A. Vega-Moreno, T. Viera-Curbelo, R. Vignaga, Instituto de Astrofísica de Canarias (Spain); E. Martínez-Gonzalez, CSIC, Univ. de Cantabria (Spain); B. Aja, E. Artal, J. Cagigas, J. L. Cano-de-Diego, E. M. Cuerno, L. de-la-Fuente, A. Pérez, J. V. Terán, E. Villa, Univ. de Cantabria (Spain); L. Piccirillo, The Univ. of Manchester (United Kingdom); A. Lasenby, Univ. of Cambridge (United Kingdom)

$91454 U$ A PSD (position sensing device) to map the shift and tilt of the SRT secondary mirror [9145-181]

T. Pisanu, F. Buffa, R. Concu, P. Marongiu, M. Pili, S. Poppi, G. Serra, E. Urru, G. Vargiu, INAF Osservatorio Astronomico di Cagliari (Italy) 
$91454 \mathrm{~V}$ Calibrating CHIME: a new radio interferometer to probe dark energy [9145-182]

L. B. Newburgh, Univ. of Toronto (Canada); G. E. Addison, M. Amiri, The Univ. of British Columbia (Canada); K. Bandura, McGill Univ. (Canada); J. R. Bond, Canadian Institute for Theoretical Astrophysics (Canada) and Canadian Institute for Advanced Research (Canada); L. Connor, Canadian Institute for Theoretical Astrophysics (Canada) and Univ. of Toronto (Canada); J.-F. Cliche, McGill Univ. (Canada); G. Davis, M. Deng, The Univ. of British Columbia (Canada); N. Denman, Univ. of Toronto (Canada); M. Dobbs, McGill Univ. (Canada); M. Fandino, H. Fong, K. Gibbs, The Univ. of British Columbia (Canada); A. Gilbert, McGill Univ. (Canada); E. Griffin, M. Halpern, The Univ. of British Columbia (Canada); D. Hanna, McGill Univ. (Canada); A. D. Hincks, G. Hinshaw, C. Höfer, The Univ. of British Columbia (Canada); P. Klages, Univ. of Toronto (Canada) and IBM Canada (Canada); T. Landecker, Dominion Radio Astrophysical Observatory, National Research Council Canada (Canada); K. Masui, The Univ. of British Columbia (Canada) and Canadian Institute for Advanced Research (Canada); J. Mena Parra, McGill Univ. (Canada); U.-L. Pen, Canadian Institute for Theoretical Astrophysics (Canada); J. Peterson, Carnegie Mellon Univ. (United States); A. Recnik, Univ. of Toronto (Canada); J. R. Shaw, Canadian Institute for Theoretical Astrophysics (Canada); K. Sigurdson, M. Sitwell, The Univ. of British Columbia (Canada); G. Smecher, McGill Univ. (Canada); R. Smegal, The Univ. of British Columbia (Canada); K. Vanderlinde, Univ. of Toronto (Canada); D. Wiebe, The Univ. of British Columbia (Canada)

9145 4W Latest progress of LAMOST primary co-phasing experiment in NIAOT, China [9145-184] Y. Zhang, X. Cui, H. Li, J. Ni, National Astronomical Observatories, Nanjing Institute of Astronomical Optics \& Technology (China) and Key Lab. of Astronomical Optics \& Technology, Nanjing Institute of Astronomical Optics \& Technology (China); A. Li, National Astronomical Observatories, Nanjing Institute of Astronomical Optics \& Technology (China), Key Lab. of Astronomical Optics \& Technology, Nanjing Institute of Astronomical Optics \& Technology (China), and Univ. of Chinese Academy of Sciences (China); Z. Yao, X. Gong, G. Liu, Y. Wang, Y. Li, Y. Zeng, G. Zhou, National Astronomical Observatories, Nanjing Institute of Astronomical Optics \& Technology (China) and Key Lab. of Astronomical Optics \& Technology, Nanjing Institute of Astronomical Optics \& Technology (China)

9145 4X SALT segmented primary mirror: inductive edge sensors [9145-186] H. Gajjar, J. Menzies, D. Buckley, South African Astronomical Observatory (South Africa); C. Neel, P. Parbaud, S. Royet, Fogale Nanotech (France)

$91454 \mathrm{Y}$ Research progress of co-phasing inductance edge sensor for Chinese extremely large telescope [9145-187]

Y. Zhang, National Astronomical Observatories, Nanjing Institute of Astronomical Optics \& Technology (China) and Key Lab. of Astronomical Optics \& Technology, Nanjing Institute of Astronomical Optics \& Technology (China); Q. Wang, National Astronomical Observatories, Nanjing Institute of Astronomical Optics \& Technology (China), Key Lab. of Astronomical Optics \& Technology, Nanjing Institute of Astronomical Optics \& Technology (China), and Univ. of Chinese Academy of Sciences (China); Y. Li, G. Zhou, J. Ni, National Astronomical Observatories, Nanjing Institute of Astronomical Optics \& Technology (China) and and Key Lab. of Astronomical Optics \& Technology, Nanjing Institute of Astronomical Optics \& Technology (China); A. Li, National Astronomical Observatories, Nanjing Institute of Astronomical Optics \& Technology (China), Key Lab. of Astronomical Optics \& Technology, Nanjing Institute of Astronomical Optics \& Technology (China), and Univ. of 
Chinese Academy of Sciences (China); H. Li, Y. Zeng, National Astronomical Observatories, Nanjing Institute of Astronomical Optics \& Technology (China) and Key Lab. of Astronomical Optics \& Technology, Nanjing Institute of Astronomical Optics \& Technology (China)

914550 Active control of the Chinese Giant Solar Telescope [9145-191]

Y. Dai, Yunnan Observatories (China); D. Yang, National Astronomical Observatories, Nanjing Institute of Astronomical Optics \& Technology (China) and Key Lab. of Astronomical Optics \& Technology, Nanjing Institute of Astronomical Optics \& Technology (China); Z. Jin, Z. Liu, Yunnan Observatories (China); W. Qin, Yunnan Association for Science and Technology (China)

$914551 \quad$ An innovative telescope control system architecture for SST-GATE telescopes at the CTA Observatory [9145-192]

G. Fasola, S. Mignot, P. Laporte, Observatoire de Paris à Meudon, CNRS, Univ. Paris Diderot (France); A. Abchiche, G. Buchholtz, Institut National des. Sciences de I'Univers, CNRS (France); I. Jégouzo, Observatoire de Paris à Meudon, CNRS, Univ. Paris Diderot (France)

914552 TCS software for the SONG telescope [9145-193]

H. Wang, C. Ren, Nanjing Institute of Astronomical Optics and Technology (China)

914553 A new telescope control system for the Telescopio Nazionale Galileo: I- derotators [9145-194]

A. Ghedina, M. Gonzalez, H. Perez Ventura, C. Carmona, A. L. Riverol, INAF - Fundación Galileo Galilei (Spain)

914554 System identification and interval analysis of the Green Bank Telescope structure and servo system [9145-195]

T. Ranka, M. Garcia-Sanz, Case Western Reserve Univ. (United States); T. Weadon, J. Ford, National Radio Astronomy Ctr. (United States)

914555 An iterative model-based cogging compensator for the Green Bank Telescope servo system [9145-196]

T. Franke, Case Western Reserve Univ. (United States); T. Weadon, J. Ford, National Radio Astronomy Observatory (United States); M. Garcia-Sanz, Case Western Reserve Univ. (United States)

914557 Keck telescope mount control redesign to improve short move performance [9145-198] P. M. Thompson, Systems Technology, Inc. (United States); T. Krasuski, K. Tsubota, J. Johnson, W. M. Keck Observatory (United States)

914558 Seismic analysis of the 4-meter telescope SST-GATE for the Cherenkov Telescope Array [9145-200]

J.-L. Dournaux, J.-M. Huet, J.-P. Amans, D. Dumas, Observatoire de Paris, CNRS, Univ. Paris Diderot (France); S. Blake, Durham Univ. (United Kingdom); H. Sol, Observatoire de Paris, CNRS, Univ. Paris Diderot (France)

914559 Two-motor direct drive control for elevation axis of telescope [9145-201] T. Tang, Y. Tan, G. Ren, Institute of Optics and Electronics (China) and The Key Lab. of Beam Control (China) 
9145 5A Simple modeling of hydrostatic bearings [9145-202]

C. Hull, Giant Magellan Telescope Organization Corp. (United States)

9145 5D Design of the planets telescope structure [9145-205]

S. Griffin, M. Edwards, J. Kuhn, Univ. of Hawai'i (United States)

9145 5E The Giant Magellan telescope (GMT): Gregorian instrument rotator bearing [9145-206]

S. Gunnels, Paragon Engineering (United States)

9145 5G Structural analysis and modifications to the V. M. Blanco telescope for dark energy camera (DECam) installation [9145-208]

P. Schurter, A. Montané, R. Tighe, T. M. C. Abbott, F. Muñoz, Cerro Tololo Inter-American

Observatory (Chile); D. Neill, National Optical Astronomy Observatory (United States)

Author Index

xxxiv 


\title{
Conference Committee
}

\author{
Symposium Chairs
}

Gillian S. Wright, UK Astronomy Technology Centre (United Kingdom)

Luc Simard, National Research Council Canada (Canada)

Symposium Co-chairs

Colin Cunningham, UK Astronomy Technology Centre

(United Kingdom)

Masanori lye, National Astronomical Observatory of Japan (Japan)

Conference Chairs

Larry M. Stepp, Thirty Meter Telescope Observatory Corporation

(United States)

Roberto Gilmozzi, European Southern Observatory (Germany)

Helen J. Hall, SOFIA / USRA (United States)

Conference Program Committee

Matthew Colless, Research School of Astronomy \& Astrophysics, The

Australian National University (Australia)

Jean-Gabriel Cuby, Laboratoire d'Astrophysique de Marseille

(France)

Xiangqun Cui, Nanjing Institute of Astronomical Optics \& Technology (China)

Frank W. Kan, Simpson Gumpertz \& Heger Inc. (United States)

Victor L. Krabbendam, LSST (United States)

Jeffrey R. Kuhn, University of Hawai'i (United States)

Heather K. Marshall, National Solar Observatory (United States)

Göran Sandell, SOFIA / USRA (United States)

Jason Spyromilio, European Southern Observatory (Germany)

Tomonori Usuda, National Astronomical Observatory of Japan

(Japan)

\section{Session Chairs}

1 Project Reviews I

Larry M. Stepp, Thirty Meter Telescope Observatory Corporation (United States)

2 Project Reviews II

Helen J. Hall, SOFIA / USRA (United States) 
3 Upgrades to Existing Observatories

Frank W. Kan, Simpson Gumpertz \& Heger Inc. (United States)

4 Site Characterization and Testing

Larry M. Stepp, Thirty Meter Telescope Observatory Corporation (United States)

5 Design of Telescopes for Extreme Environments

Xiangqun Cui, Nanjing Institute of Astronomical Optics \& Technology (China)

6 Telescopes to Image Orbiting Objects

Helen J. Hall, SOFIA / USRA (United States)

7 Cherenkov Telescopes I

Jean-Gabriel Cuby, Laboratoire d'Astrophysique de Marseille (France)

8 Cherenkov Telescopes II

Helen J. Hall, SOFIA / USRA (United States)

9 Airborne Telescopes I

Helen J. Hall, SOFIA / USRA (United States)

10 Airborne Telescopes II

Maureen L. Savage, SOFIA / USRA (United States)

11 Airborne Telescopes III

Maureen L. Savage, SOFIA / USRA (United States)

12 Telescopes and Arrays for Surveys, Time-domain and Transient Observations I

Victor L. Krabbendam, LSST (United States)

13 Telescopes and Arrays for Surveys, Time-domain and Transient Observations II

Matthew Colless, The Australian National University (Australia)

14 Telescopes and Arrays for Surveys, Time-domain and Transient Observations III

Tomonori Usuda, National Astronomical Observatory of Japan (Japan)

15 Telescope Performance Measurement

Tomonori Usuda, National Astronomical Observatory of Japan (Japan) 
16 Extremely Large Telescopes I

Jason Spyromilio, European Southern Observatory (Germany)

17 Extremely Large Telescopes II

Tomonori Usuda, National Astronomical Observatory of Japan (Japan)

18 Enabling Technologies for Extremely Large Telescopes I

Larry M. Stepp, Thirty Meter Telescope Observatory Corporation (United States)

19 Enabling Technologies for Extremely Large Telescopes II

Jeffrey R. Kuhn, Institute for Astronomy, University of Hawai'i (United States)

20 Telescope Controls

Jeffrey R. Kuhn, Institute for Astronomy, University of Hawai'i (United States)

21 Segmented Mirror Alignment, Phasing and Wavefront Control Larry M. Stepp, Thirty Meter Telescope Observatory Corporation (United States)

22 Millimeter Wavelength Telescopes and Arrays I

Jean-Gabriel Cuby, Laboratoire d'Astrophysique de Marseille (France)

23 Millimeter Wavelength Telescopes and Arrays II

Heather K. Marshall, National Solar Observatory (United States)

24 Radio Telescopes and Arrays

Xiangqun Cui, Nanjing Institute of Astronomical Optics \& Technology (China)

25 Solar Telescopes I

Heather K. Marshall, National Solar Observatory (United States)

26 Solar Telescopes II

Jason Spyromilio, European Southern Observatory (Germany)

27 AIV, Commissioning and Early Operations

Jason Spyromilio, European Southern Observatory (Germany)

28 Telescope Structures, Bearings and Drives

Victor L. Krabbendam, LSST (United States) 
29 Observatory Facilities and Enclosures

Frank W. Kan, Simpson Gumpertz \& Heger Inc. (United States)

30 Measurement and Control of Telescope Vibration

Jason Spyromilio, European Southern Observatory (Germany)

xxxviii

Proc. of SPIE Vol. 9145 914501-38

Downloaded From: https://www.spiedigitallibrary.org/conference-proceedings-of-spie on 26 Apr 2023 Terms of Use: https://www.spiedigitallibrary.org/terms-of-use 


\section{Introduction}

The large number of submissions to and excellent attendance at the Groundbased and Airborne Telescopes $V$ conference reflects continued strong interest in the astronomical and engineering communities. There were 214 papers submitted to this year's conference, which included 30 oral sessions and two poster sessions.

Good progress was reported on many ongoing and planned programs. Excellent papers were presented on AKA, ALMA, APF, AST3, BLASTPol, CCAT, CGST, CHIME, CLST, CTA, DCT, DECam, DKIST, E-ELT, GLT, GMT, HET, KDUST, LAMOST, LBT, LMT, LSST, LT2, MSE, NST, OAJ, Pan-STARRS, PLANETS, SKA, SOFIA, Spider, SST, TAO, TESS, TMT, and WFT.

Papers were presented on many current optical-IR telescope projects that are finally funded and ready for construction. The Extremely Large Telescopes session was the most widely attended; almost at the level of the conference's plenary sessions. There were several sessions of Airborne Telescopes, including SOFIA and balloon projects. Another topic that had several sessions was Telescopes and Arrays for Surveys, Time-domain and Transient Observations. Attendance was strong through the last session on Measurement and Control of Telescope Vibration.

The range of technical subjects covered in the papers is similar to previous conferences. Design of telescopes for extreme environments still is a hot topic for the conference. A new topic that hasn't been covered in the past was telescopes to image orbiting objects. This will be of interest in the future for commercial and military applications.

The co-chairs would like to thank the SPIE symposium organizers, the GB\&AT program committee members, the session chairs, the authors, and all the conference participants for making this year's conference so successful.

\section{Larry M. Stepp \\ Roberto Gilmozzi Helen J. Hall}


Proc. of SPIE Vol. $9145914501-40$

Downloaded From: https://www.spiedigitallibrary.org/conference-proceedings-of-spie on 26 Apr 2023 Terms of Use: https://www.spiedigitallibrary.org/terms-of-use 